\title{
BRANCH STRUCTURE OF $J$-HOLOMORPHIC CURVES NEAR PERIODIC ORBITS OF A CONTACT MANIFOLD
}

\author{
ADAM HARRIS AND KRZYSZTOF WYSOCKI
}

\begin{abstract}
Let $M$ be a three-dimensional contact manifold, and $\tilde{\psi}: D \backslash$ $\{0\} \rightarrow M \times \mathbb{R}$ a finite-energy pseudoholomorphic map from the punctured disc in $\mathbb{C}$ that is asymptotic to a periodic orbit of the contact form. This article examines conditions under which smooth coordinates may be defined in a tubular neighbourhood of the orbit such that $\tilde{\psi}$ resembles a holomorphic curve, invoking comparison with the theory of topological linking of plane complex algebroid curves near a singular point. Examples of this behaviour, which are studied in some detail, include pseudoholomorphic maps into $\mathbb{E}_{p, q} \times$ $\mathbb{R}$, where $\mathbb{E}_{p, q}$ denotes a rational ellipsoid (contact structure induced by the standard complex structure on $\mathbb{C}^{2}$ ), as well as contact structures arising from non-standard circle-fibrations of the three-sphere.
\end{abstract}

\section{INTRODUCTION}

The local theory of pseudoholomorphic maps from a Riemann surface into an almost complex, symplectic $4-$ manifold $(\mathcal{M}, J, \omega)$ is developed largely around comparisons with the classical theory of plane algebroid curves. Following the initial investigations of Gromov, in the works of McDuff [12], Micallef and White [14], and Sikorav [15], methods of construction of local diffeomorphisms between neighbourhoods of $p \in \mathcal{M}^{2 k}$ and $\mathbf{0} \in \mathbb{C}^{k}$ (specifically, $k=2$ ) were found in order to exhibit singular $J$-holomorphic curves $\tilde{\psi}:(\mathbb{C}, 0) \rightarrow(\mathcal{M}, p)$ as being locally equivalent to holomorphic ones. As a result the local topological data associated with singularities of plane curves can be transferred to the pseudoholomorphic context. Recall that the germ of an algebraic curve $\Gamma$ with singularity at $\mathbf{0} \in \mathbb{C}^{2}$ of multiplicity $n$ is represented by the vanishing locus of a function $f\left(w_{1}, w_{2}\right) \in \mathcal{O}_{\mathbb{C}^{2}}(\mathcal{U})$ for some neighbourhood $\mathcal{U}$ of the origin, such that the homogeneous polynomial $H_{n}\left(w_{1}, w_{2}\right)$ of terms of minimal degree $n$ in the Taylor expansion of $f$ at $\mathbf{0}$, has $n \geq 2$. Each linear factor of $H_{n}$ describes a complex tangent line to $\Gamma$ at $\mathbf{0}$, hence in order that the germ of $\Gamma$ be irreducible at the origin it is necessary that $H_{n}$ be the $n$-th power of a single linear term. If $\mathbb{S}_{\varepsilon}^{3}$ bounds a ball of radius $\varepsilon$ in $\mathcal{U}$, then the geometric locus of this term (i.e., ignoring multiplicity) intersects $\mathbb{S}_{\varepsilon}^{3}$ in a great circle $C$ corresponding to the axis of a solid torus in which $\Gamma \cap \mathbb{S}_{\varepsilon}^{3}$ also describes the trajectory of an iterated torus knot $K_{\Gamma}$. The topological "linking" of $K_{\Gamma}$ with $C$ provides a set of numerical invariants in addition to the multiplicity, on which the classification of singular curve-germs is based (cf., e.g., [2]).

Received by the editors July 18, 2005 and, in revised form, June 1, 2006.

2000 Mathematics Subject Classification. Primary 32Q65, 53D10. 
The existence of a local analytic parametrization of $\Gamma$ is a problem first studied systematically by Newton, and later developed rigorously by Weierstrass and Puiseux among others. Thus $\Gamma$ may be represented in standard form as the image of a holomorphic map $\Phi:(D, 0) \rightarrow(\mathcal{U}, \mathbf{0}), D \subseteq \mathbb{C}$, such that $\Phi(z)=\left(z^{n}, \Sigma_{i \geq n+1} a_{i} z^{i}\right)$ (cf. [2]). This type of series expansion takes advantage of the natural splitting of $\mathbb{C}^{2}$ into a product of coordinate planes. In order to conveniently see the relationship between certain essential exponents of the parametrization and the linking invariants of $K_{\Gamma}$, most treatments therefore replace the ball bounded by $\mathbb{S}_{\varepsilon}^{3}$ with a bidisc $\Delta \times \Delta$ (this is, after all, homeomorphic to the ball; hence topological data are preserved). On the other hand, the totality of all complex 1-dimensional subspaces of $\mathbb{C}^{2}$ induces a fibration of $\mathbb{S}^{3}$ by great circles, discovered by Hopf. Given a vector $\boldsymbol{v}_{p} \in \mathbb{C}^{2}$ corresponding to $p \in \mathbb{S}^{3}$, the standard complex structure $J_{0}$ defines a vector field $X(p):=J_{0} \cdot \boldsymbol{v}_{p}$ which is tangent to the great circle through each $p \in \mathbb{S}^{3}$. Via the Euclidean inner product on $\mathbb{R}^{4} \approx \mathbb{C}^{2}$, the orthogonal complement of $X$ in $T \mathbb{S}^{3}$ defines a non-integrable plane-field, or "contact structure", on $\mathbb{S}^{3}$ corresponding to the kernel of the 1 -form $\lambda_{0}$ which is the metric dual of $X$. The splitting of $\left.T \mathbb{C}^{2}\right|_{\mathbb{S}^{3}}$ induced by this structure provides an alternative natural frame for the relationship between $K_{\Gamma}$ and the holomorphic parametrization of plane curves, though we will return to this matter only in the final section as the "classical model" of a more general study.

Contact structures are defined on manifolds $M$ of odd dimension, though for the purposes of this article we will always assume the dimension to be three. If $\lambda$ is a 1 -form representing such a structure, the extension of $\omega:=d \lambda$ to $M \times \mathbb{R}$ determines a "symplectisation" of $(M, \lambda)$. Conversely, compact symplectic manifolds $(\mathcal{M}, \omega)$ with ends of symplectisation type motivate the role of contact geometry within modern symplectic topology. Through the work of Hofer, Wysocki and Zehnder [8], [9] and Eliashberg [3], $J$-holomorphic curves have been adapted via symplectisation to contact geometry and topology, specifically as a tool for analysing the Weinstein conjecture [6] and conversely for explicit construction of moduli of pseudoholomorphic maps into manifolds $\mathcal{M}$ with symplectisation-type ends [3], [9]. Recall that the "Reeb vector field" $X$ associated with a given structure $\lambda$ is uniquely determined by the conditions $\lambda(X) \equiv 1, \mathcal{L}_{X} \lambda=0$ (Lie symmetry). The Weinstein conjecture asserts that the Reeb flow of a compact contact 3-manifold always admits at least one closed characteristic (or "periodic orbit") $\gamma: \mathbb{S}^{1} \rightarrow M$ such that $\dot{\gamma}(t)=X(\gamma(t))$. If $\xi \subset T M$ denotes the plane-field corresponding to $\operatorname{ker}(\lambda)$, then a contact structure on $M$ is said to be "pseudohermitian" when it is equipped with a partial almost complex structure $j \in C^{\infty}\left(M, \xi \otimes \xi^{*}\right)$ such that $\left.d \lambda(*, j \cdot *)\right|_{\xi}$ is a positive definite and symmetric quadratic form. The splitting of $T M$ via $X$ and $\xi$ also provides a natural extension of $j$ to $T(M \times \mathbb{R})$ in relation to which the Cauchy-Riemann equation is defined for pseudoholomorphic maps into $M \times \mathbb{R}$ (cf., e.g., [6]).

Let $\tilde{\psi}: \Sigma \rightarrow M \times \mathbb{R}$ be one such map. For any smooth function $h: \mathbb{R} \rightarrow[0,1]$ we may extend the contact form to $M \times \mathbb{R}$ by defining

$$
\lambda_{h}(p, a)=h(a) \cdot \lambda(p)
$$

to act on each $T_{(p, a)}(M \times \mathbb{R})$. In particular, if $\mathcal{F}_{+}^{1}$ denotes the space of all smooth $h$ such that the derivative $h^{\prime} \geq 0$, then the "energy" is given as

$$
E(\tilde{\psi})=\sup _{h \in \mathcal{F}_{+}^{1}} \int_{\Sigma} \tilde{\psi}^{*} d \lambda_{h} .
$$


If $\psi$ denotes the projection of $\tilde{\psi}$ onto $M$ and $a$ its projection onto $\mathbb{R}$, recall that

$$
\psi^{*} d \lambda=\left(\left|\psi_{\eta}\right|^{2}+\left|\psi_{\zeta}\right|^{2}\right) d \eta \wedge d \zeta
$$

where $z=\eta+\boldsymbol{i} \zeta$ is a local complex coordinate on $\Sigma$. $E(\tilde{\psi})$ consequently vanishes if and only if $\tilde{\psi}$ is constant - a condition that follows automatically from Stokes' theorem when $\Sigma$ is compact; hence pseudoholomorphic maps into symplectisations are naturally defined on punctured Riemann surfaces. Specifically, let $\tilde{\psi}$ be a finiteenergy map defined on a punctured disc $D \backslash\{0\} \subset \mathbb{C}$ and let $r=-\ln (|z|)$ and $\varphi=\arg (z) \in \mathbb{S}^{1} \approx \mathbb{R} / 2 \pi \mathbb{Z}$. A theorem of Hofer, Wysocki and Zehnder [8] states that if $\tilde{\psi}$ has unbounded image in $M \times \mathbb{R}$, then there exists a real number $T \neq 0$ and a Reeb-periodic orbit $\gamma: \mathbb{S}^{1} \rightarrow M$ such that

$$
\lim _{r \rightarrow \infty} \psi(r, \varphi)=\gamma(T \varphi) ; \lim _{r \rightarrow \infty} \frac{a(r, \varphi)}{r}=T \text { in } C^{\infty}\left(\mathbb{S}^{1}\right)
$$

$T$ is moreover an integer multiple of the minimal period $\tau$ of $\gamma$ and corresponds to the "charge" $\lim _{r \rightarrow \infty} \frac{1}{2 \pi} \int_{\mathbb{S}^{1}} \psi^{*} \lambda$ of $\tilde{\psi}$ at $0 \in D$. Closed characteristics of the Reeb flow are thus realised asymptotically by cylinders mapped pseudoholomorphically into the corresponding symplectisation of $M$ (we pass over the very substantial theory devoted to existence of such mappings of cylinders in general; cf., however, $[6],[8])$.

The asymptotic relations established in this theorem and refined elsewhere [10] are fundamental to the present article in which we examine the topological behaviour of $\tilde{\psi}(D \backslash\{0\})$ within a tubular neighbourhood of $\mathcal{P}:=\gamma\left(\mathbb{S}^{1}\right) \subset M$. Before summarising our method and results, however, it should be mentioned that they require added technical hypotheses to be imposed locally upon the Reeb flow itself. Let $\Delta \times(\mathbb{R} / \tau \mathbb{Z})\left(\Delta \subseteq \mathbb{R}^{2}\right)$ be the tubular domain on which a general system of ordinary differential equations $\dot{\boldsymbol{x}}=f(\boldsymbol{x})$, for $f$ smooth and $\vartheta$-periodic (minimal period $\tau)$ in the coordinates $\boldsymbol{x}=(x, y, \vartheta) \in \Delta \times \mathbb{S}^{1}$, is defined. Suppose that $\{x=y=0\}$ corresponds to a periodic solution $\gamma(\vartheta)$, i.e., if $\phi(x, y, \vartheta)$ denotes the associated $\vartheta$-parametric family of diffeomorphisms, depending on initial conditions $(x, y) \in \Delta$ suffciently small, then $\gamma(\vartheta)=\phi(0,0, \vartheta), \gamma(\vartheta+\tau)=\gamma(\vartheta)$. The linear variational equation for $\dot{\boldsymbol{x}}=f(\boldsymbol{x})$ at $\gamma$ then has the form

$$
\frac{\partial}{\partial \vartheta} \phi_{*}(0,0, \vartheta)=f_{*}(0,0, \vartheta) \cdot \phi_{*}(0,0, \vartheta)
$$

noting that $f_{*}(0,0,0)=f_{*}(0,0, \tau)$ and $\phi_{*}(0,0, \tau)=A \cdot \phi_{*}(0,0,0)=A$, where $A$ denotes the "holonomy matrix" associated with $(\dagger)$. From the theory of ordinary differential equations (cf., e.g., [5]) it is well-known that the eigenvalues, or "characteristic multipliers" of $A$ are important in determining the stability of the flow $\phi$ near an orbit $\mathcal{P}$. In particular, 1 is automatically a characteristic multiplier in the $\vartheta$-direction, while eigenvalues of complex modulus greater than or less than one imply that the flow will be either unstable or asymptotically stable along closed surfaces containing $\mathcal{P}$ (cf. [5]). Specifically when $f$ corresponds to the Reeb vector field of a contact structure, the flow $\phi$ is area-preserving, hence $\operatorname{det}(A)=1$ and the remaining pair of eigenvalues are mutually reciprocal (mutually conjugate if they are unimodular). Hofer, Wysocki and Zehnder actually use the asumption that $A$ is not the identity (cf. "non-degeneracy" of $\mathcal{P}$ ) as a technical hypothesis in obtaining their asymptotic formulae (cf. [8]). From the viewpoint of the present article this condition has the disadvantage that it does not hold for the Reeb flow of the standard contact structure on the 3-sphere, corresponding to the Hopf fibration as 
mentioned above. In fact, it does not hold for a large sub-class of the contact structures which are examined below, although an extension of their methods does allow these authors to include some contact manifolds that are foliated by periodic orbits (cf. [10]). The asymptotics have been rederived in a recent thesis of F. Bourgeois [1], however, by means of the following alternative hypothesis.

Definition 1 (cf. [1]). A contact form $\lambda$ on $M$ is said to be of "Morse-Bott type" if, for every $T>0, N_{T}:=\{p \in M \mid \phi(p, T)=p\}$ is a smooth, closed, orientable submanifold of $M$ such that $\left.d \lambda\right|_{N_{T}}$ has locally constant rank, and $T_{p} N_{T}=\operatorname{ker}\left(\phi_{*}(p, T)-I d\right)$.

For present purposes it will henceforth be assumed that all contact structures under consideration are of Morse-Bott type. Our aim is to understand the extent to which $J$-holomorphic cylinders in symplectisations actually link with the periodic orbits which they approach asymptotically. In this sense the case of algebroid knots $K_{\Gamma}$ linking with great circles in the 3 -sphere suggests a classical model, since $\mathbb{S}^{3} \times \mathbb{R}$ is clearly diffeomorphic to $\mathbb{C}^{2} \backslash\{0\}$ (we will return to this matter below). By analogy with the work of Sikorav and others we will show that a suitably constructed diffeomorphism applied to a tubular neighbourhood of $\mathcal{P}$ allows $\tilde{\psi}$ to be represented locally by a holomorphic parametrization, and the sense in which this is possible will be the subject of sections two and three. The problem of "asymptotic similarity" addressed here has a global aspect, however, not found in the study of singularities of $J$-holomorphic curves. Because of this we have imposed two further conditions on the pseudohermitian structure:

(1) in a sense to be made precise in section three, $\mathcal{P}$ will be said to be "locally recurrent" if a sufficiently small disc transverse to $\mathcal{P}$ can be considered a surface of section of the Reeb flow (the diffeomorphism of the disc consequently induced by the return map will be denoted by $\alpha$ ). In particular this condition forces the characteristic multipliers of $A$ to be unimodular, but is a stronger assumption than orbital stability near $\mathcal{P}$;

(2) it will be assumed that the Reeb flow is not only a Lie symmetry of the contact form, but also the almost complex structure, i.e., $\mathcal{L}_{X} j=0$ (hence in particular $\alpha$ is diffeomorphically equivalent to a rotation).

It now remains to summarise our results.

Theorem 1 (cf. Section 3). Let $(\psi, a): D \backslash\{0\} \rightarrow M \times \mathbb{R}$ be a J-holomorphic curve of finite energy and charge $n$ at $z=0$, asymptotic to a locally recurrent periodic orbit $\mathcal{P}$, near which $\mathcal{L}_{X_{\lambda}} j=0$. Consider any tubular neighbourhood of $\mathcal{P}$ in $M$, diffeomorphic to $\Delta \times \mathbb{S}^{1}$ such that $\{0\} \times \mathbb{S}^{1} \approx \mathcal{P}$ and such that $\psi^{-1}\left(\Delta \times\left\{\vartheta_{0}\right\}\right)$ for some fixed $\vartheta_{0} \in \mathbb{S}^{1}$ divides $D \backslash\{0\}$ into $n$ "quasi-sectors" $Q_{k}$. Then there exists a diffeomorphic change of coordinates in $\Delta \times[0,2 \pi)$ such that on each $Q_{k} \subset D \backslash\{0\}$ the map $(\psi, a)$ can be expressed in the form

$$
\left(F_{k}(z), H_{k}(z)-\frac{1}{2 \pi i} \hat{G}(z, \bar{z})\right), \quad 0 \leq k \leq n-1,
$$

where $F_{k}, H_{k}$ are holomorphic on $Q_{k}$ and continuous on $\bar{Q}_{k}$, such that

$$
\left.F_{k}\right|_{\bar{Q}_{k} \cap \bar{Q}_{k+1}}=\left.\alpha \circ F_{k+1}\right|_{\bar{Q}_{k} \cap \bar{Q}_{k+1}},
$$

while each $H_{k}$ corresponds to an analytic branch of $\frac{1}{2 \pi i} \log (\rho)$ for $\rho$ holomorphic such that $\operatorname{ord}_{0}(\rho)=n$. Moreover, the function $\hat{G}$ belongs to $C^{2}(D)$ and is bounded 
by $K|z|$. Finally, if $\alpha=1$, then each $F_{k}$ is the restriction of a single function $F$ holomorphic on $D, F(0)=0$.

A representation corresponding to the classical local parametrization of plane algebroid curves is moreover easily derived when $\alpha=1$. In this case the relationship between charge and algebraic multiplicity also becomes explicit.

In section four we construct a class of pseudohermitian structures on the 3sphere for which $\alpha=1$, containing the standard structure as a distinct special case, and using results on characterisation of generalised circle fibrations due to Gluck and Warner [4]. Recent ideas of McKay [13] relating elliptic line congruences to "osculating" almost complex structures associated with a four-dimensional real vector space $V$ are also incorporated in the construction.

Theorem 2 (cf. Section 4). Let $J$ be the osculating complex structure of an elliptic line congruence $\Sigma \subset \mathbb{G} r_{2}(V)$, such that the associated skew-symmetric 2-form $\omega_{\Sigma}$ on $V \backslash\{0\}$ is closed. Then the 1 -form $\lambda$, such that $\lambda_{\boldsymbol{v}}:=i_{\boldsymbol{v}} \omega_{\Sigma}$, defines a (fillable, hence tight) contact structure on $\mathbb{S}^{3}$ for which $\left.\mathcal{L}_{X_{\lambda}} J\right|_{k e r(\lambda)}=0$ and $\alpha=1$. Moreover, two such structures are equivalent via a diffeomorphism $\delta$ of $\mathbb{S}^{3}$ if and only if $\delta \in \mathbb{O}(4)$.

(The notions of "tight" and "over-twisted" contact structures are important in the study of global dynamics and topology (cf. [3], [6]) but are not referred to explicitly in this article.)

The final section is devoted to two examples, the first of which is the standard structure on $\mathbb{S}^{3}$. Holomorphic maps $\Phi:(D, 0) \rightarrow\left(\mathbb{C}^{2}, \mathbf{0}\right)$ corresponding to irreducible germs of a curve $\Gamma$ are translated explicitly into pseudoholomorphic maps $\Psi: D \rightarrow \mathbb{S}^{3} \times \mathbb{R}$ such that the tangent to $\Gamma$ at the origin becomes the locally recurrent periodic orbit $\mathcal{P}$ corresponding to a great circle. We note that $\alpha=1$ in this case as a direct consequence of the Hopf fibration restricted to any tubular neighbourhood of $\mathcal{P}$. It should also be mentioned that the identification of algebraic curves with finite-energy pseudoholomorphic maps into $\left(\mathbb{S}^{3} \times \mathbb{R}, \lambda_{0}, J_{0}\right)$ has been addressed from a different perspective in an article of Hofer and Kriener [7]. The second example discussed in this section includes the well-known contact structures $\lambda_{0}$ more generally induced by $J_{0}$ and the Euclidean inner product on rational ellipsoids

$$
\mathbb{E}_{p, q}=\left\{\left.\left(w_{1}, w_{2}\right) \in \mathbb{C}^{2}|p| w_{1}\right|^{2}+q\left|w_{2}\right|^{2}=1\right\},
$$

where $\frac{p}{q} \in \mathbb{Q}$. In this case the hypersurface is foliated by recurrent periodic orbits such that either $\alpha$ is the identity or a non-trivial rational rotation through either $2 \pi \frac{p}{q}$ or $2 \pi \frac{q}{p}$. Given a tubular neighbourhood $\Delta \times \mathbb{S}^{1}$ of one of the two periodic orbits $\mathcal{P}$ for which $\alpha$ is non-trivial, we construct a solid torus $\Delta^{\prime} \times \mathbb{S}^{1}$ with contact form $\lambda$, and a smooth covering map $\beta: \Delta \times \mathbb{S}^{1} \rightarrow \Delta^{\prime} \times \mathbb{S}^{1}$ such that $\lambda_{0}=\beta^{*} \lambda$ and $\beta_{*} \circ j_{0}=j_{0}^{\prime} \circ \beta_{*}$ (where $j_{0}=\left.J_{0}\right|_{k e r\left(\lambda_{0}\right)}$ and $\left.j_{0}^{\prime}=\left.J_{0}\right|_{k e r(\lambda)}\right)$. Pseudoholomorphic maps asymptotic to this orbit in $\Delta \times \mathbb{S}^{1}$ then project pseudoholomorphically via $\beta$ into $\Delta^{\prime} \times \mathbb{S}^{1}$, where the central axis is a recurrent orbit of the projected Reeb vector field such that $\alpha^{\prime}=1$. Working backwards from a holomorphic parametrization in $\Delta^{\prime} \times \mathbb{S}^{1} \times \mathbb{R}$, we then explicitly construct pseudoholomorphic maps $(\psi, a): D \backslash\{0\} \rightarrow$ $\Delta \times \mathbb{S}^{1} \times \mathbb{R}$ according to the prescription of Theorem 1 (cf. section 5 ).

The authors would like to thank Dr. S. Gadde and Dr. Y. Tonegawa for helpful and stimulating discussions on occasions during the period of research for this article. The first author also gratefully acknowledges the support of an Australian 
Research Council grant during the initial phase of work undertaken at the University of Melbourne.

\section{LOCAL MODEL OF PARAMETRIZATION}

In this section we will first review the phenomenon of holomorphic similarity in the neighbourhood of a smoothly embedded point, using this as a model for the asymptotic case to follow. Let $M$ be a closed, oriented three-manifold with contact form $\lambda$ and associated Reeb vector field $X_{\lambda}$. If $\xi \subset T M$ denotes the sub-bundle corresponding to the kernel of $\lambda$, let $J$ be an almost complex structure on $\xi$ which is compatible with $\lambda$. Consider a Riemann surface $\Sigma$ and a pseudoholomorphic map

$$
(\psi, a): \Sigma \rightarrow M \times \mathbb{R} .
$$

Let $p_{0} \in i m(\psi)$ be a smoothly embedded point, and let $D$ be a neighbourhood of $\psi^{-1}\left(p_{0}\right)$ with complex coordinate $z=\eta+\boldsymbol{i} \zeta$ such that $z\left(\psi^{-1}\left(p_{0}\right)\right)=0$. For all $p \in M$ and all $v \in T_{p} M$, consider the projection map $\pi_{p}: T_{p} M \rightarrow \xi_{p}$ defined by $\pi_{p}(v)=v-\lambda_{p}(v) \cdot X_{\lambda}$. Then $\psi$ satisfies the equations

$$
\begin{gathered}
\pi\left(\psi_{\eta}\right)+J \pi\left(\psi_{\zeta}\right)=0, \\
\lambda\left(\psi_{\zeta}\right)=-a_{\eta} \quad \lambda\left(\psi_{\eta}\right)=a_{\zeta} .
\end{gathered}
$$

We recall as a consequence that

$$
\psi^{*} d \lambda=\left(\left|\pi\left(\psi_{\eta}\right)\right|^{2}+\left|\pi\left(\psi_{\zeta}\right)\right|^{2}\right) d \eta \wedge d \zeta,
$$

hence $X_{\lambda}\left(p_{0}\right)$ does not belong to the image of $\psi_{*}$ at the origin of $D$ if and only if $\left|\pi\left(\psi_{\eta}\right)\right|^{2}+\left|\pi\left(\psi_{\zeta}\right)\right|^{2}$ does not vanish there - a fact which is in turn granted by the assumption that $p_{0}$ is smoothly embedded. Now choose a system of Darboux coordinates $\left(x_{1}, x_{2}, x_{3}\right)$ neighbouring $p_{0}$ in $M$. Within this neighbourhood the image of $\psi$ may then be realized as the graph of a smooth function $x_{3}=f\left(x_{1}, x_{2}\right)$. Noting that $\lambda=d x_{3}+x_{1} d x_{2}$ and $X_{\lambda}=\frac{\partial}{\partial x_{3}}$ in these coordinates, we remark that

$$
\pi_{p}\left(v_{1}, v_{2}, v_{3}\right)=\left(v_{1}, v_{2},-x_{1} v_{2}\right) ;
$$

hence the standard projection $\left(v_{1}, v_{2}, v_{3}\right) \mapsto\left(v_{1}, v_{2}\right)$ defines a linear isomorphism $\mu$ between $\xi_{p}$ and $\mathbb{R}^{2}$ for all $p$ near the origin at $p_{0}$. It follows that $\mu \circ J \circ \mu^{-1}$ corresponds to a $2 \times 2$ matrix $j$, the restriction of which to the graph of $i m(\psi)$ depends only on the coordinates $\boldsymbol{x}:=\left(x_{1}, x_{2}\right)$. Under projection by $\mu$, equation $(*)$ then becomes

$$
\boldsymbol{x}_{\eta}(\eta, \zeta)+j(\boldsymbol{x}) \cdot \boldsymbol{x}_{\zeta}(\eta, \zeta)=\mathbf{0} .
$$

Letting $\boldsymbol{e}_{1}$ denote the vector $(1,0)$, consider the system of ordinary differential equations

$$
\frac{d \boldsymbol{x}}{d t}=j(\boldsymbol{x}) \cdot \boldsymbol{e}_{1}
$$

and define a local diffeomorphism $\varphi$ near $p_{0}$, via existence and uniqueness of solutions, such that

$$
\left(\dot{x}_{1}, \dot{x}_{2}\right)=j\left(x_{1}, x_{2}\right) \cdot e_{1} \Rightarrow \varphi(x, t):=\left(x_{1}(t), x_{2}(t)\right), \quad \varphi(x, 0)=(x, 0) .
$$

Hence

$$
\varphi_{*}^{-1} \circ j \circ \varphi_{*}=j_{0}=\left(\begin{array}{cc}
0 & -1 \\
1 & 0
\end{array}\right),
$$

and $\tilde{\boldsymbol{x}}_{\eta}:=\varphi_{*}^{-1}\left(\boldsymbol{x}_{\eta}\right), \tilde{\boldsymbol{x}}_{\zeta}:=\varphi_{*}^{-1}\left(\boldsymbol{x}_{\zeta}\right)$ implies

$$
\tilde{\boldsymbol{x}}_{\eta}(\eta, \zeta)+j_{0} \cdot \tilde{\boldsymbol{x}}_{\zeta}(\eta, \zeta)=\mathbf{0} .
$$


Or, more simply, writing $w=x+i$, we have $w=F(z)$ on a possibly smaller disc $D^{\prime} \subseteq D \subset \mathbb{C}$, containing the origin, such that $F(0)=0, \frac{\partial F}{\partial \bar{z}}=0$.

Within the coordinate neighbourhood defined by $\left(x, t, x_{3}\right)$, we now revisit the equations $(\dagger)$, with respect to

$$
\lambda^{\prime}=(\varphi \times 1)^{*} \lambda=d x_{3}+f_{1}(x, t) d x+f_{2}(x, t) d t,
$$

in the form

$$
\begin{gathered}
\lambda^{\prime}\left(\Psi_{\eta}\right)=\left(x_{3}\right)_{\eta}+f_{1}(x, t) x_{\eta}+f_{2}(x, t) t_{\eta}=a_{\zeta}, \\
\lambda^{\prime}\left(\Psi_{\zeta}\right)=\left(x_{3}\right)_{\zeta}+f_{1}(x, t) x_{\zeta}+f_{2}(x, t) t_{\zeta}=-a_{\eta},
\end{gathered}
$$

where $\Psi:=(\varphi \times 1)^{-1} \psi$. Hence $u=x_{3}+\boldsymbol{i} a$ implies

$$
\begin{gathered}
\frac{\partial u}{\partial \bar{z}}=-\left\{f_{1}(x, t) \frac{\partial x}{\partial \bar{z}}+f_{2}(x, t) \frac{\partial t}{\partial \bar{z}}\right\} \\
=-\left\{f_{1}(F(z), \bar{F}(z)) \frac{\partial}{\partial \bar{z}} \Re(F)+f_{2}(F(z), \bar{F}(z)) \frac{\partial}{\partial \bar{z}} \Im(F)\right\} \\
=-\frac{1}{2}\left[\left(f_{1}+\boldsymbol{i} f_{2}\right) \circ F(z)\right] \cdot \overline{F^{\prime}(z)}:=-F^{*} \omega,
\end{gathered}
$$

where $\omega:=\frac{1}{2}\left(f_{1}(w, \bar{w})+\boldsymbol{i} f_{2}(w, \bar{w})\right) d \bar{w}$. Let $\Delta$ denote a small disc centred at the origin of the $(x, t)$-plane, and apply the Cauchy-Green formula to obtain a smooth function $g(w, \bar{w}), g(0)=0$ satisfying the equation $\bar{\partial} g=\omega$ on $\Delta$. Then we have

$$
F^{*} \omega=\bar{\partial}\left(F^{*} g\right)=\bar{\partial}(g \circ F) .
$$

Hence for the parametrization of $\Psi$ we now have the system of equations

$$
w=F(z) ; \quad u=H(z)-g \circ F(z),
$$

with respect to holomorphic functions $F$ and $H$, which simplifies under the coordinate transformation

$$
w^{\prime}=w ; \quad u^{\prime}=u+g(w, \bar{w})-\boldsymbol{i} a(0)
$$

to $u^{\prime}=H(z)-\boldsymbol{i} a(0)$. Finally, $\operatorname{ord}_{0}(H-\boldsymbol{i} a(0))=n$ implies there exists a holomorphic function $h(z), h(0)=0, h^{\prime}(0) \neq 0$, on a possibly smaller disc $D^{\prime} \subseteq D$, such that $H-\boldsymbol{i} a(0)=h^{n}$; hence $z^{\prime}:=h(z)$ implies

$$
w^{\prime}=F \circ h^{-1}\left(z^{\prime}\right) ; \quad u^{\prime}=\left(z^{\prime}\right)^{n},
$$

which corresponds to the classical local parametrization of algebroid curves in $\mathbb{C}^{2}$. In the following section we will examine a class of periodic orbits near which the analytic representation of the local model above can be achieved in a similar manner.

\section{Asymptotic APPRoximation NeAR A PERIODiC ORBIT}

Consider a periodic orbit of the Reeb flow, denoted $\mathcal{P}$, and a tubular neighbourhood $T_{\mathcal{P}} \subset M$. If $\Delta$ represents a disc centred at the origin in $\mathbb{R}^{2}$, let $\tilde{\Delta} \subset M$ be an embedded image such that the origin is mapped to the unique element $p_{0}$ of $\mathcal{P} \cap \tilde{\Delta}$, with $\tilde{\Delta}$ itself corresponding to a transverse slice of $T_{\mathcal{P}}$. The Reeb flow will be assumed moreover to be Lyapunov-stable near $\mathcal{P}$ in the sense that for all $p \in \tilde{\Delta}^{\prime}$, where $\Delta^{\prime} \subseteq \Delta$ is a sufficiently small disc centred at the origin, there exists a unique solution $\gamma_{p}:[0, \infty) \rightarrow M$ to the equation

$$
\frac{d \gamma_{p}}{d t}=X_{\lambda}\left(\gamma_{p}(t)\right), \quad \gamma_{p}(0)=p
$$


which depends smoothly on both $t$ and $p$, and remains inside $T_{\mathcal{P}}$ for all $t \geq 0$. Given $p \in \tilde{\Delta}^{\prime}$, we will define $(i) \tau(p)$ to be the smallest $t>0$ such that $\gamma_{p}(t) \in$ $\tilde{\Delta},($ ii $) \Gamma_{p}:=\gamma_{p}((0, \tau(p)])$, and for each connected open neighbourhood of the origin $\Omega \subseteq \tilde{\Delta}$,

$$
\text { (iii) } \Gamma(\Omega):=\bigcup_{p \in \Omega \times\left\{\theta_{0}\right\}} \Gamma_{p}
$$

We may now consider a recursively defined system of neighbourhoods $\left\{\Omega_{k}\right\}$, such that $\Omega_{0}:=\tilde{\Delta}^{\prime}$, while $\Omega_{k}$ denotes the origin-component of $\Gamma\left(\Omega_{k-1}\right) \cap \Omega_{k-1}$. The set $\Omega_{\infty}:=\bigcap_{k=0}^{\infty} \Omega_{k}$ measures an important dynamic aspect of the Reeb flow.

Proposition 1. If $\Omega_{\infty}$ is open, then it is conformally equivalent to a disc.

Proof. Conformal equivalence to a disc will follow immediately if $\Omega_{\infty}$ can be shown to be simply connected, which we now prove by induction: $\Omega_{0}=\tilde{\Delta}^{\prime}$. Suppose $\Omega_{k}$ is simply connected, and let $C \subset \Omega_{k+1}$ be a simple closed loop. By the existence and uniqueness of ordinary differential equations $C$ can be traced back under the Reeb flow to a simple closed loop $C^{\prime}$ which bounds a contractible subdomain, say $\mathcal{U} \subset \Omega_{k}$. Once again, existence and uniqueness ensures that for all $p \in \mathcal{U}$ we have $\Gamma_{p} \cap \Gamma\left(C^{\prime}\right)=\emptyset$, while the Jordan Curve Theorem for plane domains implies that the domain bounded by a given closed simple loop is unique. It follows that

$$
C=\Gamma(\partial \mathcal{U}) \cap \Omega_{k}=\partial \Gamma(\mathcal{U}) \cap \Omega_{k}=\partial\left(\Gamma(\mathcal{U}) \cap \Omega_{k}\right) .
$$

Moreover, $\Gamma(\mathcal{U}) \cap \Omega_{k}$ is a homeomorphic image of $\mathcal{U}$, and is therefore itself a contractible subdomain, hence $\Omega_{k+1}$ is simply connected.

Now consider a simple closed loop $C \subset \Omega_{\infty}$, i.e., $C \subset \Omega_{k}$ for all $k$, and hence there exists a contractible subdomain $\mathcal{U}_{k}$ in $\Omega_{k}$ such that $\partial \mathcal{U}_{k}=C$. But uniqueness of the interior of $C$ of course implies that $\mathcal{U}_{k} \equiv \mathcal{U}$ for all $k$, and hence $\mathcal{U} \subset \Omega_{\infty}$.

Definition 2. The Reeb flow will be said to be "locally recurrent" near a periodic orbit $\mathcal{P}$ if it is Lyapunov-stable within a tubular neighbourhood $T_{\mathcal{P}}$ and for any sufficiently small embedded disc $\tilde{\Delta}$, corresponding to a transversal slice through $T_{\mathcal{P}}$ at some point $p_{0}$, the limit set $\Omega_{\infty} \subseteq \tilde{\Delta^{\prime}} \subseteq \tilde{\Delta}$ is open. An orbit $\mathcal{P}$ itself may also be referred to as "locally recurrent" in this context.

As mentioned in section 1 , the treatment of a finite-energy pseudoholomorphic map $(\psi, a): \Sigma \rightarrow M \times \mathbb{R}$ from a punctured Riemann surface, asymptotic to a periodic orbit, may be restricted to a map between $D \backslash\{0\} \subset \mathbb{C}$ and, for convenience, the Martinet neighbourhood $\Delta \times \mathbb{S}^{1} \times\left[a_{0}, \infty\right)$ of $\mathcal{P}$, with coordinates $(x, y, \theta, a)$ (cf. [8], [10], [11]). In particular, for $p_{0}$ corresponding to the origin of $\Delta$, we have $\gamma_{0}(t)=\left(0,0, e^{\boldsymbol{i}\left(\theta_{0}+2 \pi t / \tau_{0}\right)}\right)$, where $\tau_{0}$ denotes the minimal period of $\mathcal{P}$. In relation to the asymptotic results of [8], it will sometimes be convenient to use cylindrical coordinates on $D \backslash\{0\}$, viz. $(r, \varphi)=(-\ln (|z|)$, arg $(z)) \in\left[r_{0}, \infty\right) \times \mathbb{S}^{1}$. In particular, the charge of the puncture is given by

$$
T:=\frac{1}{2 \pi} \lim _{r \rightarrow \infty} \int_{\mathbb{S}^{1}} \psi^{*} \lambda,
$$

and is a well-defined integer multiple of $\tau_{0}$ (cf. [8]). Within this coordinate system, the asymptotic relations of Hofer, Wysocki and Zehnder (mentioned in section one) 
may be interpreted as

$$
\begin{gathered}
a(r, \varphi)=T r+a_{0}+\varepsilon(r, \varphi), \\
\theta(r, \varphi)=T\left(\frac{\varphi}{\tau_{0}}\right)+\theta_{0}+\delta(r, \varphi),
\end{gathered}
$$

with $\varepsilon, \delta$ approaching zero uniformly in $\varphi$ as $r \rightarrow \infty$. For $|z|$ sufficiently small (i.e., $r$ sufficiently large) we may think of $\theta^{-1}\left(\theta_{0}\right)$ as a union of radial arcs meeting at the origin and differing by only a small perturbation from the rays defined by $\arg (z)=\frac{1}{n}\left(\theta_{0}+2 \pi k\right), 0 \leq k \leq n-1$, where $n=\frac{T}{\tau_{0}}$. Hence we will consider $D \backslash\{0\}$ as a union of $n$ quasi-sectors $Q_{k}$, bounded by these arcs, on which the quasi-analytic "branches" of $(\psi, a)$ are defined.

For the local model in section 2 it was sufficient to assume that $\psi$ is a local embedding in order to represent the image as a graph on which the restriction of an almost complex structure $J$ depends on just two of the coordinates of a Darboux chart. Under the assumption that the Reeb flow is locally recurrent near $\mathcal{P}$, we now select $\Omega_{\infty} \times\left\{\theta_{0}\right\}$ as a coordinate disc within the initial Martinet tube (on which $\lambda=f \cdot(d \vartheta+x d y)$, for a function $f$ such that $f(0,0, \vartheta) \equiv \tau_{0}$ and $\nabla f(0,0, \vartheta) \equiv \mathbf{0}$ as described in [8], [11], though these facts are not used here). Without loss of generality, let $\theta_{0}$ be zero and consider the cylinder $\Omega_{\infty} \times[0,2 \pi]$, which maps to the tube via the obvious identification $\bmod (2 \pi)$. The cylinder has $\mathcal{P}$ as its axis, $x=y=0$, and the Reeb vector field in Martinet coordinates already looks like $\frac{1}{\tau_{0}} \frac{\partial}{\partial \theta}$ when restricted to $\mathcal{P}$. There is no consequent loss of generality if we "normalise" $\lambda$ by the constant multiple $\frac{1}{\tau_{0}}$, so that the minimal period is effectively 1 , and hence the charge $T$ is an integer. By analogy with the standard construction of Darboux coordinates, the next step is to define

$$
\mathcal{C}:=\left\{(p, t) \mid p=(x, y) \in \Omega_{\infty}, \quad 0 \leq t \leq \tau(p)\right\}
$$

and a homeomorphism

$$
h: \mathcal{C} \rightarrow \Omega_{\infty} \times[0,2 \pi],\left.\quad h\right|_{\Omega_{\infty} \times\{0\}}=1,
$$

which is smooth for all $0<t<\tau(p)$, coming from solutions of the ordinary differential equation

$$
\frac{d \gamma_{p}}{d t}=X_{\lambda}\left(\gamma_{p}(t)\right)
$$

It follows that on the interior of $\mathcal{C}$, the standard contact form $\lambda_{0}$ and $\lambda^{\prime}:=h^{*} \lambda$ have the same Reeb vector field, corresponding to $\frac{\partial}{\partial t}$. We now consider the CauchyRiemann system

$$
\begin{gathered}
\pi\left(\left(h^{-1} \psi\right)_{\eta}\right)+J \pi\left(\left(h^{-1} \psi\right)_{\zeta}\right)=0, \\
\lambda^{\prime}\left(\left(h^{-1} \psi\right)_{\zeta}\right)=-a_{\eta} \quad \lambda^{\prime}\left(\left(h^{-1} \psi\right)_{\eta}\right)=a_{\zeta} .
\end{gathered}
$$

As in the local model, for a sufficiently "thin" neighbourhood of $\mathcal{P}$, the standard projection $\left(v_{1}, v_{2}, v_{3}\right) \mapsto\left(v_{1}, v_{2}\right)$ determines a linear isomorphism $\mu$ between $\xi^{\prime}:=$ $\operatorname{ker}\left(\lambda^{\prime}\right)$ and $\mathbb{R}^{2}$. Hence we define a $2 \times 2$ matrix-valued function $j(x, y)=\mu \circ J \circ \mu^{-1}$, such that $\boldsymbol{x}:=(x, y)$ implies $(* *)$ can be written in the form

$$
\boldsymbol{x}_{\eta}(z)+j \boldsymbol{x}_{\zeta}(z)=0 .
$$

Let $\alpha$ denote the diffeomorphism of $\Omega_{\infty} \times\left\{\theta_{0}\right\}$ defined by the return map $\alpha(p):=$ $\gamma_{p}(\tau(p))$, hence $\alpha(0)=0$. 
Lemma 1. If $\mathcal{L}_{X_{\lambda}} J=0$, then in a neighbourhood of $0 \in \Delta$, the smooth automorphism $\alpha$ is diffeomorphically equivalent to a rotation.

Proof. Letting $\boldsymbol{e}_{1}$ denote the vector $(1,0)$, consider the system of ordinary differential equations

$$
\frac{d \boldsymbol{x}}{d s}=j(\boldsymbol{x}) \cdot \boldsymbol{e}_{1}
$$

and define a local diffeomorphism $\varphi: \Delta^{\prime \prime} \rightarrow U \subseteq \Omega_{\infty}$ via existence and uniqueness of solutions, such that

$$
\left(\dot{x}_{1}, \dot{x}_{2}\right)=j(\boldsymbol{x}) \cdot \boldsymbol{e}_{1} \Rightarrow \varphi(x, s):=\left(x_{1}(s), x_{2}(s)\right),
$$

$\varphi(x, 0)=(x, 0)$. Hence

$$
\varphi_{*}^{-1} \circ j \circ \varphi_{*}=j_{0}=\left(\begin{array}{cc}
0 & -1 \\
1 & 0
\end{array}\right) .
$$

Let $\Omega_{\infty}^{\prime}$ denote the simply connected domain inside $U$ which is stabilised by the Reeb flow. The diffeomorphism $\hat{\alpha}:=\varphi^{-1} \circ \alpha \circ \varphi$ then acts on $\varphi^{-1}\left(\Omega_{\infty}^{\prime}\right) \subseteq$ $\Delta^{\prime \prime}$ as a restricted automorphism such that $\hat{\alpha}(0)=0$ under the assumption of local recurrence. The additional assumption $\mathcal{L}_{X_{\lambda}} J=0$ implies that $\alpha^{*} j=j$, hence in particular $\hat{\alpha} j_{0}=j_{0} \hat{\alpha}$, i.e., $\hat{\alpha}$ is a conformal automorphism. Modulo a conformal transformation identifying $\varphi^{-1}\left(\Omega_{\infty}^{\prime}\right)$ with a disc, $\hat{\alpha}$ is then equivalent to a rotation.

Now $\tilde{\boldsymbol{x}}_{\zeta}:=\varphi_{*}^{-1}\left(\boldsymbol{x}_{\zeta}\right)$ and $\tilde{\boldsymbol{x}}_{\eta}:=\varphi_{*}^{-1}\left(\boldsymbol{x}_{\eta}\right)$ implies

$$
\tilde{\boldsymbol{x}}_{\eta}(\eta, \zeta)+j_{0} \cdot \tilde{\boldsymbol{x}}_{\zeta}(\eta, \zeta)=\mathbf{0}
$$

Note that each "branch" of

$$
\Psi:=(h \circ(\varphi \times 1))^{-1} \psi
$$

is defined smoothly in the interior and continuously up to the boundaries of a quasisector $Q_{k}$ in $D \backslash\{0\}$, but discontinuities arise at points $z_{0}$ lying on the smooth arcs that bound adjacent sectors (in the usual way " \pm " will be used to denote opposite sides of the boundary). Discontinuities of the transverse projection of $\Psi$ are therefore described by the relations

$$
\lim _{z \rightarrow z_{0}^{ \pm}} \tilde{\boldsymbol{x}}(z):=\tilde{\boldsymbol{x}}^{ \pm}\left(z_{0}\right) \Rightarrow \hat{\alpha}\left(\tilde{\boldsymbol{x}}^{-}\left(z_{0}\right)\right)=\tilde{\boldsymbol{x}}^{+}\left(z_{0}\right) .
$$

Hence on each $Q_{k} \subset D \backslash\{0\}$, (†*) defines a holomorphic function $w=F_{k}(z)$ which partially describes a branch of $\Psi$, such that

$$
\left.F_{k}\right|_{\bar{Q}_{k} \cap \bar{Q}_{k+1}}=\left.\hat{\alpha} \circ F_{k+1}\right|_{\bar{Q}_{k} \cap \bar{Q}_{k+1}}, \quad 0 \leq k \leq n-1 .
$$

Lemma 2. If the Reeb flow determines a fibration of a tubular neighbourhood by periodic orbits over $\Omega_{\infty}$, i.e., $\alpha=1$, then there is a single holomorphic function $F(z)$ on $D, F(0)=0$, describing the transverse projection of $\Psi$.

Proof. $\alpha=1$ implies the existence of a function $F(z)$, continuous on $D$ and holomorphic on the interior of each quasi-sector. The demonstration that $F$ is holomorphic on $D$ is a standard application of Morera's Theorem (and the Removable Singularities Theorem at the origin). Specifically, let $z_{0}$ lie on one of the smooth arcs bounding a quasi-sector and let $z^{\prime}=\eta^{\prime}+\boldsymbol{i} \zeta^{\prime}$ be a local complex coordinate with respect to which the arc is locally described as the graph of a function $\zeta^{\prime}=\rho\left(\eta^{\prime}\right)$. Let $\Gamma$ be a simple loop inside the $z^{\prime}$-coordinate neighbourhood of $z_{0}$. Clearly if $\Gamma$ 
does not intersect the arc, then $F$ must be holomorphic on a slightly larger domain containing $\Gamma$, hence

$$
\int_{\Gamma} F d z^{\prime}=0
$$

If $\Gamma$ intersects the arc, consider a simple affine coordinate transformation so that the cord joining the endpoints $a, b$ of the intersection is now the axis corresponding to $\zeta^{\prime}=0$. Hence, without loss of generality, we may assume $\rho(a)=\rho(b)=0$. Let $\gamma$ denote the arc corresponding to the graph between $a$ and $b$, and for $\varepsilon>0$ let $\gamma_{ \pm \varepsilon}$ denote the arcs corresponding to $\zeta^{\prime}=\rho\left(\eta^{\prime}\right) \pm \varepsilon$, lying between $a \pm \boldsymbol{i} \varepsilon$ and $b \pm \boldsymbol{i} \varepsilon$ and on either side of $\gamma$. From the continuity of $F$ it follows that

$$
\begin{gathered}
\int_{\gamma} F d z^{\prime}=\int_{a}^{b} F\left(\eta^{\prime}+\boldsymbol{i} \rho\left(\eta^{\prime}\right)\right) d \eta^{\prime}=\lim _{\varepsilon \rightarrow 0} \int_{a}^{b} F\left(\eta^{\prime}+\boldsymbol{i}\left(\rho\left(\eta^{\prime}\right) \pm \varepsilon\right)\right) d \eta^{\prime} \\
=\lim _{\varepsilon \rightarrow 0} \int_{\gamma_{\varepsilon}} F d z^{\prime} .
\end{gathered}
$$

Now decomposing $\Gamma$ into two simple loops $\Gamma_{ \pm}$having $\gamma$ as their common boundary component, and applying the above limit, we see that

$$
\int_{\Gamma} F d z^{\prime}=0
$$

Hence $F$ extends holomorphically to a neighbourhood of $z_{0}$.

Now returning to the particular form of the equations $(\dagger \dagger)$ in $\mathcal{C}$ note that any $\lambda^{\prime}$ with $\frac{\partial}{\partial t}$ as its Reeb vector field must take the general form

$$
\lambda^{\prime}=d t+f_{1}(\boldsymbol{x}) d x+f_{2}(\boldsymbol{x}) d y .
$$

As was seen with respect to a Darboux chart of the local model, such a local presentation of the contact form allows decoupling of $(\dagger \dagger)$ into an inhomogeneous Cauchy-Riemann equation. This property is preserved under the diffeomorphism $\varphi$, however, if it is assumed that $\mathcal{L}_{X_{\lambda}} J=0$, hence in particular the matrix $j$ above is independent of $t$. In this case, letting $u=t+\boldsymbol{i} a$, (††) becomes

$$
\begin{aligned}
\frac{\partial}{\partial \bar{z}}\left[\left.u\right|_{Q_{k}}\right] & =-\left\{f_{1}\left(F_{k}(z)\right) \frac{\partial \Re\left(F_{k}\right)}{\partial \bar{z}}+f_{2}\left(F_{k}(z)\right) \frac{\partial \Im\left(F_{k}\right)}{\partial \bar{z}}\right\} \\
& =-\frac{1}{2}\left[\left(f_{1}+\boldsymbol{i} f_{2}\right) \circ F_{k}(z)\right] \cdot \overline{F_{k}^{\prime}(z)}
\end{aligned}
$$

keeping in mind that this equation is defined smoothly only on the interior of each quasi-sector $Q_{k}$. Define $\omega:=\frac{1}{2}\left(f_{1}+\boldsymbol{i} f_{2}\right)(w, \bar{w}) d \bar{w}$, so that

$$
\begin{gathered}
\lambda^{\prime}=d t+2 \Re(\omega), \text { and } \\
\frac{1}{2}\left[\left(f_{1}+\boldsymbol{i} f_{2}\right) \circ F_{k}(z)\right] \cdot \overline{F_{k}^{\prime}(z)} d \bar{z}=F_{k}^{*} \omega .
\end{gathered}
$$

Now $\mathcal{L}_{X_{\lambda}} \lambda=0$ implies $\hat{\alpha}^{*} \Re(\omega)=\Re(\omega)$. In particular, f $:=\left(f_{1}, f_{2}\right)$ implies $\Re(\omega)=(\mathbf{f}, *)$ with respect to the standard inner product on $\mathbb{R}^{2}$, and hence $\hat{\alpha}_{*}^{t} \mathbf{f}=\mathbf{f}$. Similarly $\Im(\omega)=\left(j_{0} \mathbf{f}, *\right)$, while $\mathcal{L}_{X_{\lambda}} J=0$ implies $\hat{\alpha}_{*}^{t} j_{0}=j_{0} \hat{\alpha}_{*}^{t}$, so that

$$
\hat{\alpha}^{*} \Im(\omega)=\left(\hat{\alpha}_{*}^{t} j_{0} \mathbf{f}, *\right)=\left(j_{0} \mathbf{f}, *\right)=\Im(\omega) .
$$


It follows that $\hat{\alpha}^{*} \omega=\omega$, and hence

$$
\begin{gathered}
\left.F_{k}^{*} \omega\right|_{\bar{Q}_{k} \cap \bar{Q}_{k+1}}=\left.F_{k}^{*}\left(\hat{\alpha}^{*} \omega\right)\right|_{\bar{Q}_{k} \cap \bar{Q}_{k+1}}=\left.\left(\hat{\alpha} \circ F_{k}\right)^{*} \omega\right|_{\bar{Q}_{k} \cap \bar{Q}_{k+1}} \\
=\left.F_{k+1}^{*} \omega\right|_{\bar{Q}_{k} \cap \bar{Q}_{k+1}} .
\end{gathered}
$$

There now exists a continuous function $G(z, \bar{z})$ on $D$ such that

$$
\left.G(z, \bar{z}) d \bar{z}\right|_{Q_{k}}:=F_{k}^{*} \omega, \quad 0 \leq k \leq n-1 .
$$

Letting $\Phi(w, \bar{w})=f_{1}+\boldsymbol{i} f_{2}$ and assuming, from Lemma 1 , that $\hat{\alpha}$ is a rotation, we may write

$$
\hat{\alpha}^{*} \omega=\omega \Rightarrow \Phi \circ \hat{\alpha}(w, \bar{w})=\overline{\hat{\alpha}}^{-1} \Phi(w, \bar{w}) .
$$

It is easily seen that

$$
\Phi_{w} \circ \hat{\alpha}(w, \bar{w})=|\hat{\alpha}|^{-2} \Phi_{w}, \quad \Phi_{\bar{w}} \circ \hat{\alpha}(w, \bar{w})=\overline{\hat{\alpha}^{-2}} \Phi_{\bar{w}},
$$

and hence

$$
\begin{gathered}
\left.\frac{\partial G}{\partial z}\right|_{\bar{Q}_{k} \cap \bar{Q}_{k+1}}=\Phi_{w}\left(F_{k+1}\right) \cdot\left|F_{k+1}^{\prime}\right|^{2} \\
=\Phi_{w}\left(\hat{\alpha} \cdot F_{k}\right) \cdot\left|\hat{\alpha} \cdot F_{k}^{\prime}\right|^{2}=\Phi_{w}\left(F_{k}\right) \cdot\left|F_{k}^{\prime}\right|^{2},
\end{gathered}
$$

while

$$
\begin{gathered}
\left.\frac{\partial G}{\partial \bar{z}}\right|_{\bar{Q}_{k} \cap \bar{Q}_{k+1}}=\Phi_{\bar{w}}\left(F_{k+1}\right) \cdot\left(\bar{F}_{k+1}^{\prime}\right)^{2}+\Phi\left(F_{k+1}\right) \cdot \bar{F}_{k+1}^{\prime \prime} \\
=\Phi_{\bar{w}}\left(\hat{\alpha} \cdot F_{k}\right) \cdot\left({\overline{\hat{\alpha} \cdot F_{k}^{\prime}}}^{\prime}\right)^{2}+\Phi\left(\hat{\alpha} \cdot F_{k}\right) \cdot \overline{\hat{\alpha} \cdot F_{k}^{\prime \prime}} \\
=\Phi_{\bar{w}}\left(F_{k}\right) \cdot\left(\bar{F}_{k}^{\prime}\right)^{2}+\Phi\left(F_{k}\right) \cdot \bar{F}_{k}^{\prime \prime} .
\end{gathered}
$$

Thus $G$ is a continuously differentiable function, and

$$
\hat{G}(z, \bar{z}):=\int_{D} \frac{G(\mu, \bar{\mu})}{\mu-z} d \mu \wedge d \bar{\mu}
$$

is a twice-continuously differentiable function on $D$. We may therefore write the solutions $u=H_{k}(z)-\frac{1}{2 \pi i} \hat{G}(z, \bar{z})$ for a holomorphic function $H_{k}$ defined on each $Q_{k}$. Now $a(z)=\Im(u)$ is a smooth function on $D \backslash\{0\}$, while $\hat{G}$ belongs to $C^{2}(D)$. Hence $\Im\left(H_{k}(z)\right)$ must in fact correspond to a single function $h(z)$ for all $k$, which belongs to $C^{2}(D \backslash\{0\})$ and is harmonic inside each $Q_{k}$, therefore harmonic throughout $D \backslash\{0\}$. Moreover, the harmonic conjugate of $h$ is uniquely defined up to a constant, hence we have a single harmonic function $\hat{h}$ on the punctured disc such that

$$
\Re\left(H_{k}(z)\right)=\hat{h}(z)+c_{k}, \quad 0 \leq k \leq n-1 .
$$

Recall that $\Re(u)$ corresponds to $t$ such that $0<t<\tau(p)$ for some $(p, t)$ belonging to the image of $\Psi$. Discontinuities of $t$ along the boundaries of each $Q_{k}$ are consequently determined by $\tau(p)$, i.e.,

$$
\lim _{z \rightarrow z_{0}^{-}} \hat{h}_{k}(z)=\lim _{z \rightarrow z_{0}^{+}} \hat{h}_{k+1}(z)+\tau\left(\tilde{\boldsymbol{x}}^{-}\left(z_{0}\right)\right)
$$

for $z_{0}$ lying in the boundary arc $\bar{Q}_{k} \cap \bar{Q}_{k+1}$. From the discussion above it follows that $\tau\left(\tilde{\boldsymbol{x}}^{-}(z)\right)$ must be constant for $\Psi$ restricted to a boundary arc. By continuity of these arcs as they radiate from the origin of $D$, this constant value must correspond to $\tau_{0}$, or 1 if $\lambda$ is assumed to have been normalised. The arcs themselves were 
originally defined by the arbitrary choice of $\theta_{0} \in \mathbb{S}^{1}$, hence we may conclude that $\tau(p)=1$ for all $p \in \Omega_{\infty}^{\prime}$. Moreover, we have

$$
c_{k+1}-c_{k}=1, \quad 0 \leq k \leq n-1,
$$

where, without loss of generality, we may set $c_{0}=0$, hence $c_{k}=k$. Recalling the asymptotic formulae $a(z)=-\ln |z|^{n}+\varepsilon(z)$ and $\theta(z)=\arg \left(z^{n}\right)+\delta(z)$, one may also notice the approximation of the holomorphic part of $u$ by the branched analytic function $\log \left(z^{n}\right)$. Specifically,

$$
u=\frac{1}{2 \pi \boldsymbol{i}}\left\{\log \left(\rho(z) e^{-\hat{G}(z, \bar{z})}\right)\right\},
$$

where the analytic function $\rho(z):=e^{2 \pi H(z)}$ has order $n$ at $z=0$, and $K:=$ $\sup _{D}|G(z, \bar{z})|$ implies

$$
\frac{1}{2 \pi}|\hat{G}(z, \bar{z})|=\frac{1}{2 \pi}\left|\int_{D} \frac{G(\mu, \bar{\mu})}{\mu-z} d \mu \wedge d \bar{\mu}\right| \leq K|z|
$$

is uniformly bounded in the parameter $\arg (z)$, i.e.,

$$
\lim _{r \rightarrow \infty}|\hat{G}(r, \varphi)|=0 \quad \text { in } C^{0}\left(\mathbb{S}^{1}\right) .
$$

In summary, we have the following

Theorem 1. Let $(\psi, a): D \backslash\{0\} \rightarrow M \times \mathbb{R}$ be a J-holomorphic curve of finite energy and charge $n$ at $z=0$, asymptotic to a locally recurrent periodic orbit $\mathcal{P}$, near which $\mathcal{L}_{X_{\lambda}} J=0$. Consider any tubular neighbourhood of $\mathcal{P}$ in $M$, diffeomorphic to $\Delta \times \mathbb{S}^{1}$ such that $\{0\} \times \mathbb{S}^{1} \approx \mathcal{P}$. There exists a diffeomorphic change of coordinates in $\Delta \times[0,2 \pi)$ such that on each quasi-sector $Q_{k} \subset D \backslash\{0\}$ the map $(\psi, a)$ can be expressed in the form

$$
\left(F_{k}(z), H_{k}(z)-\frac{1}{2 \pi i} \hat{G}(z, \bar{z})\right), \quad 0 \leq k \leq n-1,
$$

where $F_{k}, H_{k}$ are holomorphic on $Q_{k}$ and continuous on $\bar{Q}_{k}$, such that

$$
\left.F_{k}\right|_{\bar{Q}_{k} \cap \bar{Q}_{k+1}}=\left.\hat{\alpha} \circ F_{k+1}\right|_{\bar{Q}_{k} \cap \bar{Q}_{k+1}},
$$

while each $H_{k}$ corresponds to an analytic branch of $\frac{1}{2 \pi i} \log (\rho), \operatorname{ord}_{0}(\rho)=n$. Moreover, the function $\hat{G}$ belongs to $C^{2}(D)$ and is bounded by $K|z|$. Finally, if $\alpha=1$, then each $F_{k}$ is the restriction of a single function $F$ holomorphic on $D, F(0)=0$.

A representation corresponding to the classical local parametrization of plane algebroid curves is easily obtained as follows for the case $\alpha=1$. Let

$$
g(w, \bar{w}):=\frac{1}{2 \pi \boldsymbol{i}} \int_{\Delta^{\prime}} \frac{\left(f_{1}+\boldsymbol{i} f_{2}\right)(\mu, \bar{\mu}) d \mu \wedge d \bar{\mu}}{\mu-w},
$$

so that $\bar{\partial} g=\omega$. Then

$$
\begin{gathered}
G(z, \bar{z}) d \bar{z}=F^{*} \omega=F^{*}(\bar{\partial} g) \\
\quad=\bar{\partial}\left(F^{*} g\right)=\bar{\partial}(g \circ F),
\end{gathered}
$$

and hence $\hat{G}=2 \pi \boldsymbol{i}(g \circ F+\hat{H})$, for some holomorphic function $\hat{H}$. Now define a coordinate

$$
v:=e^{2 \pi i u}=\rho(z) e^{-\hat{G}}=\rho(z) e^{-g \circ F-\hat{H}}
$$


and let $\rho(z) e^{-\hat{H}}=f(z)^{n}$ for some holomorphic function $f$ on $D^{\prime} \subseteq D$, with $f^{\prime}(0) \neq$ 0 . Now $\xi:=f(z)$ implies $w=F(z)=F \circ f^{-1}(\xi)$, while $v=\xi^{n} e^{-g \circ F \circ f^{-1}(\xi)}$. From the final coordinate diffeomorphism $w^{\prime}=w ; v^{\prime}=v e^{g(w, \bar{w})}$, it now follows that

$$
w^{\prime}=F \circ f^{-1}(\xi) ; \quad v^{\prime}=\xi^{n} .
$$

\section{4. $\alpha=1$ : Circle fibrations of $\mathbb{S}^{3}$}

A class of examples of tight contact structures for which $\alpha=1$ and $\mathcal{L}_{X_{\lambda}} J=0$ near a periodic orbit is provided by the circle fibrations of $\mathbb{S}^{3}$, of which the most elementary instances are the Hopf fibrations. Given a four-dimensional real vector space $V$, these fibrations correspond to families of invariant planes (i.e., "complex lines") distinguished by linear endomorphisms $J_{0}$ determining standard complex structures on $V$, and are parametrised by $\mathbb{O}(4) / \mathbb{U}(2)$. The base space of each such fibration is a (Riemann) sphere inside the Grassmann manifold $\mathbb{G} r_{2}(V)$. More general fibrations correspond to families of planes ("line congruences") parametrised by compact surfaces $\Sigma \subset \mathbb{G} r_{2}(V)$. A line congruence is said to be elliptic if for all $P \in \Sigma$, there exists a 2 -sphere $\mathbb{S}$ corresponding to some $J_{0}$ such that $T_{P} \Sigma=T_{P} \mathbb{S}$ inside $\mathbb{G} r_{2}(V)$, hence in particular $\Sigma$ is itself diffeomorphic to a sphere. Let $\bigwedge^{2} V$ denote the space of exterior 2-forms, on which the duality operator acts in the standard way. The spaces of "self-dual" and "anti-self-dual" forms then correspond to +1 and -1 eigenspaces of this operator, defining a direct sum decomposition $\bigwedge^{2} V \cong \bigwedge_{+}^{2} \oplus \bigwedge_{-}^{2}$. If $\mathbb{S}_{+}$and $\mathbb{S}_{-}$denote the 2 -spheres of radius $\frac{1}{\sqrt{2}}$ inside each of these eigenspaces, then the Grassmann manifold of oriented 2-planes of $V$ is wellknown to correspond to $\mathbb{S}_{+} \times \mathbb{S}_{-}$. Moreover, it was shown by Gluck and Warner [4] that the surfaces $\Sigma$ of generalised Hopf fibrations are precisely the graphs of distance-decreasing smooth maps $f: \mathbb{S}_{-} \rightarrow \mathbb{S}_{+}$, with standard Hopf fibrations corresponding to constant maps. In [13], McKay also observed that they correspond to general elliptic line congruences and hence determine non-linear complex structures on $V$ which "osculate" with linear structures at each $P \in \Sigma$. We apply this idea to the explicit construction of contact structures on $\mathbb{S}^{3}$ as follows. Let $\mathcal{J}(V)$ denote the space of linear endomorphisms of $V$ corresponding to linear complex structures. Then it was shown in [13] that each elliptic line congruence $\Sigma$ determines a map

$$
J: \Sigma \rightarrow \mathcal{J}(V) \subset V \otimes V^{*}
$$

such that for each plane $P \in \Sigma, J(P)$ is linear and is the "osculating" structure to $\Sigma$ at $P$ in the sense that both $P$ and $P^{\perp}$ (with respect to a given inner product on $V$ ) are complex lines relative to $J(P)$. The family of planes determined by $\Sigma$ describes a rank-two vector bundle $\mathcal{P} \stackrel{\pi}{\rightarrow} \Sigma$ such that the total space, corresponding to the incidence manifold $\mathcal{P}=\{(\boldsymbol{v}, P) \in V \times \Sigma \mid \boldsymbol{v} \in P\}$, also maps surjectively to $V$. In fact, there exists $\mathcal{P} \stackrel{\sigma}{\rightarrow} V$ such that $\mathcal{P} \backslash \sigma^{-1}(0) \cong V \backslash\{0\}$. Hence define $\varphi:=\pi \circ \sigma^{-1}: V \backslash\{0\} \rightarrow \Sigma$, so that $\varphi^{-1}(P)=\{\boldsymbol{v} \in V \backslash\{0\} \mid \boldsymbol{v} \in P\}$.

Consider the pullback $\varphi^{*} J: V \backslash\{0\} \rightarrow V \otimes V^{*}$. Hence with respect to a designated orthonormal basis of $V$, noting that $J$ and $\varphi^{*} J$ are skew-symmetric matrix-valued functions, we may represent it in the form

$$
\varphi^{*} J=\Sigma_{\mu . \nu} J^{\mu \nu} \frac{\partial}{\partial x_{\mu}} \wedge d x_{\nu} .
$$


Note moreover that the isomorphism $V^{*} \cong V$ via the Euclidean inner product allows us to define a 2-form $\omega_{\Sigma}:=\Sigma_{\mu . \nu} J^{\mu \nu} d x_{\mu} \wedge d x_{\nu}$. In the following, let $i_{v}$ denote contraction of a form by the position vector $\boldsymbol{v}=\frac{1}{2} \Sigma_{\eta} v_{\eta} \frac{\partial}{\partial \eta}$.

Lemma 3. If $d \omega_{\Sigma}=0$, then $\omega_{\Sigma}(\boldsymbol{v})=d\left(i_{\boldsymbol{v}} \omega_{\Sigma}\right)$.

Proof.

$$
\begin{aligned}
i_{\boldsymbol{v}} \omega_{\Sigma} & =\frac{1}{2} \Sigma_{\mu<\nu} J^{\mu \nu}\left(x_{\mu} d x_{\nu}-x_{\nu} d x_{\mu}\right) \\
& =\frac{1}{2} \Sigma_{\beta \neq \alpha}(-1)^{\varepsilon} x_{\beta} J^{\alpha \beta} d x_{\alpha},
\end{aligned}
$$

where

$$
\varepsilon= \begin{cases}0, & \beta<\alpha \\ 1, & \beta>\alpha\end{cases}
$$

Therefore

$$
\begin{gathered}
d\left(i_{\boldsymbol{v}} \omega_{\Sigma}\right)=\omega_{\Sigma}+\frac{1}{2} \Sigma_{\gamma<\alpha \neq \beta}(-1)^{\varepsilon} x_{\beta}\left(\frac{\partial J^{\alpha \beta}}{\partial x_{\gamma}}-\frac{\partial J^{\gamma \beta}}{\partial x_{\alpha}}\right) d x_{\gamma} \wedge d x_{\alpha} \\
=\omega_{\Sigma}+\frac{1}{2} \Sigma_{\gamma<\alpha \neq \beta} x_{\beta} \frac{\partial J^{\gamma \alpha}}{\partial x_{\beta}} d x_{\gamma} \wedge d x_{\alpha}
\end{gathered}
$$

(using the relations provided by $d \omega_{\Sigma}=0$ )

$$
=\omega_{\Sigma}+\frac{1}{2} \Sigma_{\gamma<\alpha}\left(\nabla_{\boldsymbol{v}} J^{\gamma \alpha}\right) d x_{\gamma} \wedge d x_{\alpha} .
$$

Note that the functions $J^{\gamma \alpha}$, obtained by pulling back $J$, are constant in the radial directions of $V$, hence the directional derivatives $\nabla_{\boldsymbol{v}} J^{\gamma \alpha}=0$ for all $\boldsymbol{v} \in V \backslash\{0\}$.

Now define the 1 -form $\lambda_{\Sigma}:=i_{\boldsymbol{v}} \omega_{\Sigma}$ for $\omega_{\Sigma}$ closed, noting that if $[J]$ denotes the matrix of $\varphi^{*} J$, then we may express $\lambda_{\Sigma}$ in terms of the inner product as

$$
\lambda_{\Sigma}(\boldsymbol{w})=-(\boldsymbol{w},[J] \cdot \boldsymbol{v})
$$

for all $\boldsymbol{w} \in V$. Moreover, let $X_{\lambda}$ be the vector field defined by $X_{\lambda}(\boldsymbol{v})=-[J] \cdot \boldsymbol{v}$, so that

(i) $X_{\lambda}$ is tangent to $\mathbb{S}^{3}$, since

$$
\left(\boldsymbol{v}, X_{\lambda}(\boldsymbol{v})\right)=-(\boldsymbol{v},[J] \cdot \boldsymbol{v})=-\left([J]^{t} \cdot \boldsymbol{v}, \boldsymbol{v}\right)=([J] \cdot \boldsymbol{v}, \boldsymbol{v}),
$$

hence $\left(\boldsymbol{v}, X_{\lambda}(\boldsymbol{v})\right)=0$,

(ii) $\lambda_{\Sigma}\left(X_{\lambda}\right)=|[J] \cdot \boldsymbol{v}|^{2}=1$, since $[J]_{\boldsymbol{v}} \in \mathbb{O}(4)$ for each $\boldsymbol{v} \in V \backslash\{0\}$, and

(iii) for all $\boldsymbol{w}, \boldsymbol{u} \in V$ we have

$$
\omega_{\Sigma}(\boldsymbol{w}, \boldsymbol{u})=\Sigma_{\mu<\nu} J^{\mu \nu}\left(w_{\mu} u_{\nu}-w_{\nu} u_{\mu}\right)=(\boldsymbol{w},[J] \cdot \boldsymbol{u}),
$$

and hence

$$
i_{X_{\lambda}} \omega_{\Sigma}=-\left(*,[J]^{2} \cdot \boldsymbol{v}\right)=(*, \boldsymbol{v})=0
$$

when restricted to $T \mathbb{S}^{3}$. Clearly $\varphi^{*} J$ is preserved by the Reeb flow, i.e., $\mathcal{L}_{X_{\lambda}} \varphi^{*} J=$ 0 . Moreover, if $P_{\boldsymbol{v}}$ denotes the subspace spanned by $\{\boldsymbol{v},[J] \cdot \boldsymbol{v}\}$, then $\operatorname{ker}(\lambda) \cap T \mathbb{S}^{3}=$ $P_{\boldsymbol{v}}^{\perp}$ is also an invariant subspace of $[J]_{\boldsymbol{v}}$, so we may write $j:=\left.[J]\right|_{P_{\boldsymbol{v}}^{\perp}}$ for all $\boldsymbol{v} \in \mathbb{S}^{3}$. In particular, for all $\xi \in \operatorname{ker}(\lambda)$, we have $\omega_{\Sigma}(\xi, j \cdot \xi)=-|\xi|^{2}$, which means that $\lambda_{\Sigma}$ is a contact structure compatible with the partial complex structure $j$.

It should be mentioned that all Hopf fibrations, including the non-linear ones, are smoothly equivalent as circle bundles (cf. [4]), and yet at the level of contact 
structures they are distinct. For suppose $\delta: \mathbb{S}^{3} \rightarrow \mathbb{S}^{3}$ is a diffeomorphism that identifies the Reeb flows of a given structure $\lambda_{\Sigma}$ and that of the standard structure $\lambda_{0}$. In particular, suppose that $\delta_{*} X_{\lambda}=X_{\lambda_{0}}$ and $\delta_{*} \circ \varphi^{*} J \circ \delta_{*}^{-1}=J_{0}$, where $J$ denotes the osculating complex structure associated with $\lambda_{\Sigma}$. Now assume in addition that $\delta^{*} \lambda_{0}=\lambda_{\Sigma}$, hence

$$
\delta^{*} \lambda_{0}(\boldsymbol{u})=\lambda_{0}\left(\delta_{*} \boldsymbol{u}\right)=\left(\delta_{*} \boldsymbol{u}, J_{0} \cdot \boldsymbol{v}\right)=\lambda_{\Sigma}(\boldsymbol{u})=\left(\boldsymbol{u}, \varphi^{*} J \cdot \delta_{*}^{-1} \boldsymbol{v}\right) .
$$

Therefore

$$
\left(\delta_{*} \boldsymbol{u}, J_{0} \cdot \boldsymbol{v}\right)=\left(\boldsymbol{u}, \delta_{*}^{-1} J_{0} \delta_{*} \delta_{*}^{-1} \boldsymbol{v}\right)=\left(\boldsymbol{u}, \delta_{*}^{-1} J_{0} \cdot \boldsymbol{v}\right)
$$

that is,

$$
\left(\boldsymbol{u}, \delta_{*}^{t} J_{0} \cdot \boldsymbol{v}\right)=\left(\boldsymbol{u}, \delta_{*}^{-1} J_{0} \cdot \boldsymbol{v}\right),
$$

and hence $\delta_{*}^{t} X_{\lambda_{0}}=\delta_{*}^{-1} X_{\lambda_{0}}$ (or, conversely, $\delta_{*} X_{\lambda}=\left(\delta_{*}^{t}\right)^{-1} X_{\lambda}$ ). On the other hand, $\left(\delta_{*} \boldsymbol{u}, J_{0} \cdot \boldsymbol{v}\right)=\left(\left(\delta_{*}^{-1}\right)^{t} \boldsymbol{u}, J_{0} \cdot \boldsymbol{v}\right)$ if and only if

$$
\left(\delta_{*} \boldsymbol{u}, \delta_{*} \varphi^{*} J \delta_{*}^{-1} \boldsymbol{v}\right)=\left(\left(\delta_{*}^{-1}\right)^{t} \boldsymbol{u}, \delta_{*} \varphi^{*} J \delta_{*}^{-1} \boldsymbol{v}\right),
$$

i.e., letting $\boldsymbol{v}^{\prime}:=\delta_{*}^{-1} \boldsymbol{v}$,

$$
\left(\delta_{*}^{t} \delta_{*} \boldsymbol{u}, \varphi^{*} J \cdot \boldsymbol{v}^{\prime}\right)=\left(\boldsymbol{u}, \varphi^{*} J \cdot \boldsymbol{v}^{\prime}\right) .
$$

In particular, we see that $\operatorname{ker}\left(\lambda_{\Sigma}\right)$ is an invariant subspace of $\delta_{*}^{t} \delta_{*}$. Note moreover that

$$
\delta_{*}^{t} \delta_{*} \varphi^{*} J \delta_{*}^{-1}\left(\delta_{*}^{t}\right)^{-1}=\left(\delta_{*}^{-1} J_{0}^{t} \delta_{*}\right)^{t}=\varphi^{*} J,
$$

which implies that $\left.\delta_{*}^{t} \delta_{*}\right|_{k e r(\lambda)}$ is complex-linear. But since it is clearly symmetric, it follows that $\left.\delta_{*}^{t} \delta_{*}\right|_{\operatorname{ker}(\lambda)}=c \cdot I$ for some $c \in \mathbb{R} \backslash\{0\}$. Now $d\left(\delta^{*} \lambda_{0}\right)=\delta^{*}\left(d \lambda_{0}\right)=d \lambda_{\Sigma}$ implies

$$
\begin{gathered}
d \lambda_{\Sigma}(\boldsymbol{u}, \boldsymbol{w})=d \lambda_{0}\left(\delta_{*} \boldsymbol{u}, \delta_{*} \boldsymbol{w}\right)=\left(\delta_{*} \boldsymbol{u}, J_{0} \cdot \delta_{*} \boldsymbol{w}\right) \\
=\left(\boldsymbol{u}, \delta_{*}^{t} J_{0} \delta_{*} \boldsymbol{w}\right)=\left(\boldsymbol{u}, \delta_{*}^{t} \delta_{*} \varphi^{*} J \cdot \boldsymbol{w}\right)=\left(\boldsymbol{u}, \varphi^{*} J \delta_{*}^{t} \delta_{*} \boldsymbol{w}\right)=c\left(\boldsymbol{u}, \varphi^{*} J \cdot \boldsymbol{w}\right)
\end{gathered}
$$

(if $\boldsymbol{w} \in \operatorname{ker}\left(\lambda_{\Sigma}\right)$ ), and hence

$$
d \lambda_{\Sigma}(\boldsymbol{u}, \boldsymbol{w})=c \cdot d \lambda_{\Sigma}(\boldsymbol{u}, \boldsymbol{w}), \text { i.e., } c=1 .
$$

We conclude that $\delta_{*}^{t}=\delta_{*}^{-1}$, and hence that $\delta \in \mathbb{O}(4)$, which restricts any such equivalence of contact structures to the family of linear Hopf fibrations. In summary:

Theorem 2. Let $J$ be the osculating complex structure of an elliptic line congruence $\Sigma \subset \mathbb{G} r_{2}(V)$, such that the skew-symmetric 2 -form $\omega_{\Sigma}$ on $V \backslash\{0\}$ is closed. Then the 1-form $\lambda$, such that $\lambda_{\boldsymbol{v}}:=i_{\boldsymbol{v}} \omega_{\Sigma}$, defines a fillable, hence tight, contact structure on $\mathbb{S}^{3}$ for which $\mathcal{L}_{X_{\lambda}} J=0$ and $\alpha=1$. Moreover, two such structures are equivalent via a diffeomorphism $\delta$ of $\mathbb{S}^{3}$ if and only if $\delta \in \mathbb{O}(4)$.

\section{The Classical models of the SPHere and RATional Ellipsoids}

As an explicit illustration of Theorem 1, we will first examine the classical case of analytic curves in $\mathbb{C}^{2}$. The realization of algebraic curves as finite-energy pseudoholomorphic maps has also been examined in [7], though from a slightly different point of view. Let $M=\mathbb{S}^{3} \subseteq \mathbb{C}^{2}$, with

$$
\left(\lambda_{0}\right)_{\boldsymbol{v}}=\left.\left(*, J_{0} \cdot \boldsymbol{v}\right)\right|_{T \mathbb{S}^{3}}
$$


the standard contact form defined with respect to the complex structure $J_{0}$ of $\mathbb{C}^{2}$ and the Euclidean inner product of $\mathbb{R}^{4} . M$ is then defined by the equation $\left|w_{1}\right|^{2}+\left|w_{2}\right|^{2}=1$ with respect to complex coordinates in $\mathbb{C}^{2}$, while

$$
\lambda_{0}=\Re\left(-\boldsymbol{i}\left(\bar{w}_{1} d w_{1}+\bar{w}_{2} d w_{2}\right)\right) .
$$

Consider $\Phi(z): D \rightarrow \mathbb{C}^{2}$ a complex-analytic curve defined on a neighbourhood of the origin in $\mathbb{C}$ such that $\Phi(z)=\left(z^{n}, \rho(z)\right)$ and $\operatorname{ord}_{0}(\rho) \geq n+1$, hence $\Phi$ has a singularity of multiplicity $n$ at $(0,0)$. The corresponding map $(\psi, a): D \rightarrow \mathbb{S}^{3} \times \mathbb{R}$ is given by

$$
\begin{gathered}
\psi(z)=\left[|\rho|^{2}+|z|^{2 n}\right]^{-\frac{1}{2}}\left(z^{n}, \rho(z)\right) \\
a(z)=-\frac{1}{2} \ln \left(|z|^{2 n}+|\rho|^{2}\right)=-n \ln |z|-\frac{1}{2} \ln \left(1+|z|^{-2 n}|\rho|^{2}\right) .
\end{gathered}
$$

The periodic orbit $\mathcal{P}$ corresponds simply to the circle defined by $\left\{w_{2}=0\right\} \cap \mathbb{S}^{3}$ and is associated with the degenerate tangent cone of $\Phi$ at $(0,0)$. It is, moreover, a simple calculation to verify that $\operatorname{charge}_{z=0}(\psi)=n$. Consider the proper holomorphic map $\sigma: \mathcal{O}_{\mathbb{P}_{1}}(-1) \rightarrow \mathbb{C}^{2}$, where $\mathcal{O}_{\mathbb{P}_{1}}(-1)$ denotes the complex line bundle of Chern class equal to -1 on the Riemann Sphere $\mathbb{P}_{1}(\mathbb{C})=\sigma^{-1}(\mathbf{0})$, with $\sigma: \mathcal{O}_{\mathbb{P}_{1}}(-1) \backslash \sigma^{-1}(\mathbf{0}) \cong$ $\mathbb{C}^{2} \backslash\{0\}$, given in local coordinates by the quadratic transformation $w_{1}=\mu, w_{2}=$ $\mu \nu$. Noting that $|\mu|^{2}\left(1+|\nu|^{2}\right)=1$ on the chart of $\sigma^{-1}\left(\mathbb{S}^{3}\right)$ corresponding to $\nu \neq \infty$, we have

$$
\sigma^{*} \lambda_{0}=\Re\left(-i\left(\mu^{-1} d \mu+\bar{\nu}|\mu|^{2} d \nu\right)\right) ;
$$

moreover, for each $\nu \in \mathbb{P}_{1}(\mathbb{C}) \backslash\{\infty\}$, the Hopf fibration corresponding to

$$
\varpi: \sigma^{-1}\left(\mathbb{S}^{3}\right) \rightarrow \mathbb{P}_{1}(\mathbb{C}) \approx \mathbb{S}^{2}
$$

has fibres $\varpi^{-1}(\nu)=\left\{\mu \in \mathbb{C}|| \mu \mid=\left(1+|\nu|^{2}\right)^{-\frac{1}{2}}\right\}$. Let $\nu=x+\boldsymbol{i} y$; then $\mu=|\mu| e^{\boldsymbol{i} \vartheta}=$ $\left(1+x^{2}+y^{2}\right)^{-\frac{1}{2}} e^{i \vartheta}, h:=\left.\sigma\right|_{\varpi^{-1}\left(\mathbb{P}_{1} \backslash\{\infty\}\right)}$ implies

$$
\begin{gathered}
h^{*} \lambda_{0}=\Re\left(-\boldsymbol{i}\left\{\mu^{-1} d \mu+\bar{\nu}|\mu|^{2} d \nu\right\}\right) \\
=\Re\left(-\boldsymbol{i}\left\{\left(\sqrt{1+x^{2}+y^{2}}\right) e^{\boldsymbol{i} \vartheta}\left(\frac{-(x d x+y d y) e^{\boldsymbol{i} \vartheta}}{\sqrt{\left(1+x^{2}+y^{2}\right)^{3}}}+\frac{\boldsymbol{i} e^{\boldsymbol{i} \vartheta} d \vartheta}{\sqrt{1+x^{2}+y^{2}}}\right)\right.\right. \\
\left.\left.+(x-\boldsymbol{i} y) \frac{d x+\boldsymbol{i} d y}{1+x^{2}+y^{2}}\right\}\right) \\
=\Re\left(-\boldsymbol{i}\left\{\frac{-(x d x+y d y)}{1+x^{2}+y^{2}}+\frac{x d x+y d y}{1+x^{2}+y^{2}}+\boldsymbol{i}\left(d \vartheta+\frac{x d y-y d x}{1+x^{2}+y^{2}}\right)\right\}\right) \\
=d \vartheta+\left(1+x^{2}+y^{2}\right)^{-1}(x d y-y d x),
\end{gathered}
$$

while $h^{*} X_{\lambda_{0}}=\frac{\partial}{\partial \vartheta}$. Now

$$
\Psi(z):=h^{-1} \psi(z)=\left(\arg \left(z^{n}\right), F(z)\right) \text { where } F(z):=z^{-n} \rho(z) .
$$

Clearly $\nu=F(z)$ is holomorphic, so it remains to show that

$$
h^{*} \lambda_{0}\left(\Psi_{\eta}\right)=a_{\zeta} ; h^{*} \lambda_{0}\left(\Psi_{\zeta}\right)=-a_{\eta} .
$$


Note that $a(z)=-\frac{1}{2} \ln \left(\left(\eta^{2}+\zeta^{2}\right)^{n}\left(1+x^{2}+y^{2}\right)\right)$ implies

$$
\begin{gathered}
a_{\zeta}=\frac{-n \zeta\left(\eta^{2}+\zeta^{2}\right)^{n-1}\left(1+x^{2}+y^{2}\right)-\left(\eta^{2}+\zeta^{2}\right)^{n}\left(x x_{\zeta}+y y_{\zeta}\right)}{\left(\eta^{2}+\zeta^{2}\right)^{n}\left(1+x^{2}+y^{2}\right)} \\
=\frac{-n \zeta}{\eta^{2}+\zeta^{2}}-\frac{x x_{\zeta}+y y_{\zeta}}{1+x^{2}+y^{2}},
\end{gathered}
$$

while $\Psi_{\eta}=\left(x_{\eta}, y_{\eta}, \vartheta_{\eta}\right)=\left(x_{\eta}, y_{\eta}, \frac{-n \zeta}{\eta^{2}+\zeta^{2}}\right)$ implies

$$
h^{*} \lambda_{0}\left(\Psi_{\eta}\right)=\frac{-n \zeta}{\eta^{2}+\zeta^{2}}+\frac{x y_{\eta}-y x_{\eta}}{1+x^{2}+y^{2}} .
$$

But $\nu=x+\boldsymbol{i} y=F(z)$ is holomorphic, hence $x_{\eta}=y_{\zeta}, x_{\zeta}=-y_{\eta}$ yields $a_{\zeta}=$ $h^{*} \lambda_{0}\left(\Psi_{\eta}\right)$, and similarly for $a_{\eta}$.

Recalling the discussion of section 3 , if we write $\lambda^{\prime}=d t+f_{1}(x, y) d x+f_{2}(x, y) d y$, then $h^{*} \lambda_{0}=2 \pi \lambda^{\prime}$, where

$$
f_{1}(x, y)=\frac{-y}{2 \pi\left(1+|\nu|^{2}\right)} \quad ; \quad f_{2}(x, y)=\frac{x}{2 \pi\left(1+|\nu|^{2}\right)} .
$$

Now

$$
\frac{1}{2}\left(f_{1}+i f_{2}\right)=\frac{i \nu}{4 \pi\left(1+|\nu|^{2}\right)}=\frac{i}{4 \pi} \bar{\partial} \ln \left(1+|\nu|^{2}\right),
$$

and hence $g \circ F=\frac{\boldsymbol{i}}{2 \pi} \ln \left(1+|F|^{2}\right)^{\frac{1}{2}}$. Moreover, we can simply define $\hat{G}=2 \pi \boldsymbol{i} g \circ F=$ $-\ln \left(1+|F|^{2}\right)^{\frac{1}{2}}$.

On the other hand, modulo rescaling by $2 \pi$ so that

$$
\hat{a}(z)=\frac{-1}{2 \pi} \ln \left(|z|^{n}\left(1+|F|^{2}\right)^{\frac{1}{2}}\right),
$$

we have

$$
\begin{gathered}
u=t+\boldsymbol{i} \hat{a}=\frac{1}{2 \pi}\left(\arg \left(z^{n}\right)+\boldsymbol{i}\left\{-\ln |z|^{n}-\ln \left(1+|F|^{2}\right)^{\frac{1}{2}}\right\}\right) \\
=\frac{1}{2 \pi \boldsymbol{i}}\left(\log \left(z^{n}\right)-\hat{G}\right),
\end{gathered}
$$

in accordance with the statement of Theorem 1.

Let $\Gamma \subset \mathbb{C}^{2}$ be the locus of the plane curve parametrized by $\Phi$. We remark in conclusion that although the multiplicity of the singular point of the strict transform $\overline{\sigma^{-1}(\Gamma) \backslash \sigma^{-1}(0)}$ is less than $n$, the charge at $z=0$ of $\Psi$ is easily seen to be conserved by the diffeomorphism $h$. The singular plane curve corresponding to the strict transform (assuming the singularity has not been resolved by a single quadratic transformation) is in fact asymptotic (viewed locally as a $J$-holomorphic curve $\Psi^{\prime}$ ) to a distinct periodic orbit within a new 3 -sphere bounding a neighbourhood of the transformed singularity. However, the linking of the transform of $K_{\Gamma}$ with the original periodic orbit of $\sigma^{-1}\left(\mathbb{S}^{3}\right)$ is topologically unaffected.

Now let us turn to the ellipsoids

$$
\mathbb{E}_{p, q}:=\left\{\left.\left(w_{1}, w_{2}\right) \in \mathbb{C}^{2}|p| w_{1}\right|^{2}+q\left|w_{2}\right|^{2}=1 ;(p, q) \in \mathbb{R}_{+}^{2}\right\} .
$$

The restriction to $\mathbb{E}_{p, q}$ of $\lambda_{0}$ as defined above determines a different contact structure on $T \mathbb{E}_{p, q}$, and in particular a Reeb vector field $X_{P}:=A \cdot J_{0} \cdot \boldsymbol{v}_{P}$ for all $P \in \mathbb{E}_{p, q}$, where

$$
A=\left(\begin{array}{ll}
p & 0 \\
0 & q
\end{array}\right)
$$


is viewed as an element of $\boldsymbol{G L}(2, \mathbb{C})$. Solutions of the equation $\dot{\gamma}(t)=X(\gamma(t)), \gamma(0)$ $=P=\left(z_{1}, z_{2}\right)$, then correspond to maps $t \mapsto\left(z_{1} e^{i p t}, z_{2} e^{i q t}\right)$ (cf., e.g., [7]). Note that there are two periodic orbits corresponding to $w_{1}=0$ and $w_{2}=0$ separately. These are the only periodic orbits of the Reeb flow if $\frac{p}{q}$ is irrational, whereas $\mathbb{E}_{p, q}$ is foliated by periodic orbits if $\frac{p}{q} \in \mathbb{Q}$. Moreover the periodic orbit corresponding to $w_{1}=0$ has minimal period $\tau=\frac{2 \pi}{q}$ and for $w_{2}=0$ it is $\frac{2 \pi}{p}$, while $\tau=\frac{2 \pi k}{p}=\frac{2 \pi l}{q}$ for all other orbits with respect to fixed relatively prime positive integers $k, l$. Without loss of generality, consider a tubular neighbourhood $\mathcal{U}$ of the orbit $w_{2}=0$, and let $\nu:=\frac{z_{2}^{k}}{z_{1}^{l}}$ such that $l>k$. All periodic orbits of the Reeb vector field within this tubular neighbourhood then correspond to intersections of $\mathbb{E}_{p, q}$ with algebraic curves (uniquely determined by $\nu$ ) of the form

$$
w_{2}^{k}=\nu w_{1}^{l}, \quad|\nu|<\varepsilon,
$$

for some positive $\varepsilon$. Once again, we have

$$
\lambda_{0}=\Re\left(-\boldsymbol{i}\left(\bar{w}_{1} d w_{1}+\bar{w}_{2} d w_{2}\right)\right),
$$

but this time it will be convenient to introduce a formal coordinate transformation of the form

$$
w_{1}=\mu, \quad w_{2}=\left(\nu \mu^{l}\right)^{\frac{1}{k}}
$$

so that

$$
\bar{w}_{1} d w_{1}+\bar{w}_{2} d w_{2}=\bar{\mu} d \mu+\frac{1}{k}|\nu|^{\frac{2}{k}}|\mu|^{\frac{2 l}{k}}\left(\frac{1}{\nu} d \nu+\frac{l}{\mu} d \mu\right) .
$$

Moreover, $p\left|w_{1}\right|^{2}+q\left|w_{2}\right|^{2}=1$ implies

$$
|\nu|^{\frac{2}{k}}|\mu|^{\frac{2 l}{k}}=\frac{1}{q}\left(1-p|\mu|^{2}\right) \text {, }
$$

hence

$$
\bar{w}_{1} d w_{1}+\bar{w}_{2} d w_{2}=\left(\bar{\mu}+\frac{\left(1-p|\mu|^{2}\right) l}{k q \mu}\right) d \mu+\left(\frac{1-p|\mu|^{2}}{k q \nu}\right) d \nu .
$$

Now $\mu=r e^{i \vartheta}$ implies $d \mu=e^{i \vartheta}(d r+\boldsymbol{i} r d \vartheta)$, so that

$$
\left(\bar{\mu}+\frac{\left(1-p|\mu|^{2}\right) l}{k q \mu}\right) d \mu=\frac{1}{p}\left(\frac{d r}{r}+i d \vartheta\right),
$$

while $\nu=x+i y$ implies

$$
\frac{1-p|\mu|^{2}}{k q \nu} d \nu=\frac{1-p|\mu|^{2}}{k q}\left\{\frac{x d x+y d y}{x^{2}+y^{2}}+\boldsymbol{i}\left(\frac{x d y-y d x}{x^{2}+y^{2}}\right)\right\},
$$

and hence

$$
\lambda_{0}=\frac{1}{p}\left\{d \vartheta+\frac{\left(1-p|\mu|^{2}\right)(x d y-y d x)}{l\left(x^{2}+y^{2}\right)}\right\} .
$$

Consider $f(r):=p r^{2}+q|\nu|^{\frac{2}{k}} r^{\frac{2 l}{k}}-1$, so that

$$
f^{\prime}(r)=2 p r\left(1+\frac{q^{2}}{p^{2}}|\nu|^{\frac{2}{k}} r^{2\left(\frac{l}{k}-1\right)}\right) .
$$

Hence $f^{\prime}(r)=0$ when $r=0$ or $\left(\frac{-p^{2}}{q^{2}|\nu|^{\frac{2}{k}}}\right)^{\frac{k}{2(l-k)}}$. Moreover $f(0)=-1, \lim _{r \rightarrow \infty} f(r)$ $=\infty$ implies that the equation $f(r)=0$ has a unique positive real solution. In other 
words, the value of $|\mu|$ satisfying the equation $p|\mu|^{2}+q|\nu|^{\frac{2}{k}}|\mu|^{\frac{2 l}{k}}=1$ is uniquely determined by $p, q,|\nu|$. Hence write $|\mu|=\varphi(p, q,|\nu|)$ and recall that

$$
w_{2}=\left(\nu\left(\varphi(|\nu|) e^{i \vartheta}\right)^{l}\right)^{\frac{1}{k}}, \text { i.e., } w_{2}^{k} e^{-i l \vartheta}=\nu \varphi(|\nu|)^{l} .
$$

Note also that $\varphi(p, q, 0)=\frac{1}{\sqrt{p}}$ implies that the complex function $\chi(\nu):=\nu \varphi(|\nu|)^{l}$ admits a locally differentiable inverse, and we may write

$$
\nu=\chi^{-1}\left(w_{2}^{k} e^{-i l \vartheta}\right) \text {. }
$$

Let $\Delta=\left\{\left|w_{2}\right|<\varepsilon\right\}, \Delta^{\prime}=\left\{|\nu|<\varepsilon^{\prime}\right\}$ and consider the $k$-fold covering map $\beta: \mathcal{U} \approx \Delta \times \mathbb{S}^{1} \rightarrow \Delta^{\prime} \times \mathbb{S}^{1}$ such that

$$
\beta\left(w_{2}, \vartheta\right)=\left(\chi^{-1}\left(w_{2}^{k} e^{-i l \vartheta}\right), \vartheta\right) .
$$

Alternatively, the map $\Theta: \mathbb{C}^{2} \rightarrow \mathbb{C}^{2}$ such that $(\mu, \nu)=\Theta\left(w_{1}, w_{2}\right)=\left(w_{1}, w_{2}^{k} w_{1}^{-l}\right)$ is holomorphic away from $\left\{w_{1}=0\right\}$ such that $\beta=\Theta \mid \mathbb{E}_{p, q}$. The equation (*) above may then be written more precisely in the form $\lambda_{0}=\frac{1}{p} \beta^{*} \lambda$, where

$$
\lambda=d \vartheta+\frac{\left(1-p \varphi(p, q,|\nu|)^{2}\right)(x d y-y d x)}{l|\nu|^{2}}
$$

(note also that $\lim _{|\nu| \rightarrow 0} \frac{1-p \varphi^{2}}{l|\nu|^{2}}(x d y-y d x)=0$ ). Consequently finite-energy pseudoholomorphic maps

$$
(\psi, a): D \backslash\{0\} \rightarrow \Delta \times \mathbb{S}^{1} \times \mathbb{R}
$$

project onto finite-energy maps $(\beta \circ \psi, a)$, pseudoholomorphic with respect to $\frac{1}{p} \lambda$. This claim is easily verified if we note that

$$
a_{\zeta}=\frac{1}{p} \beta^{*} \lambda\left(\psi_{\eta}\right)=\frac{1}{p} \lambda\left(\beta_{*} \circ \psi_{\eta}\right)=\frac{1}{p} \lambda\left((\beta \circ \psi)_{\eta}\right),
$$

and similarly for $a_{\eta}$. Moreover,

$$
\tilde{\pi}\left((\beta \circ \psi)_{\eta}\right):=\beta_{*} \psi_{\eta}-\frac{1}{p} \lambda\left((\beta \circ \psi)_{\eta}\right) \tilde{X},
$$

where $\tilde{X}:=\beta_{*} X_{\lambda_{0}}=p \frac{\partial}{\partial \vartheta}$ is a well-defined vector field under the above conditions. Hence

and therefore

$$
\tilde{\pi}\left((\beta \circ \psi)_{\eta}\right)=\beta_{*}\left(\pi\left(\psi_{\eta}\right)\right)
$$

$$
0=\beta_{*}\left(\pi\left(\psi_{\eta}\right)+J_{0} \pi\left(\psi_{\zeta}\right)\right)=\tilde{\pi}(\beta \circ \psi)_{\eta}+\beta_{*}\left(J_{0} \pi(\psi)_{\zeta}\right) .
$$

Let $J_{0}$ denote the standard complex structure on $\mathbb{C}^{2}$ as represented by both $\left(w_{1}, w_{2}\right)$ and $(\mu, \nu)$ (and its restriction to the contact planes of $\lambda_{0}$ and $\lambda$, respectively). Then $\beta=\left.\Theta\right|_{\mathbb{E}_{p, q}}$ for $\Theta$ holomorphic implies $\beta_{*} \circ J_{0}=J_{0} \circ \beta_{*}$, and hence

$$
0=\tilde{\pi}(\beta \circ \psi)_{\eta}+J_{0} \tilde{\pi}(\beta \circ \psi)_{\zeta} .
$$

Note that the tubular neighbourhood into which $\beta \circ \psi$ maps is fibred by Reeb orbits, hence the return map $\alpha=1$. By comparison, the Reeb flow in a neighbourhood of the original orbit $\left\{w_{2}=0\right\}$ in $\mathbb{E}_{p, q}$ induces a return map that is equivalent to a rational rotation through $2 \pi \frac{l}{k}$. In order to find a class of $J$-holomorphic curves asymptotic to the given periodic orbit within $\mathbb{E}_{p, q}$, let us first make a harmless renormalization of the contact structure, i.e., $\lambda_{0}^{\prime}:=p \cdot \lambda_{0}, X_{\lambda_{0}^{\prime}}=\frac{1}{p} X_{\lambda_{0}}$. Now

$$
\lambda_{0}^{\prime}=d \vartheta+\frac{\left(1-p \cdot \varphi(|\nu|)^{2}\right)(x d y-y d x)}{l \cdot|\nu|^{2}},
$$


and we are ready to work backwards from a holomorphic parametrization of the form

$$
\mu=z^{n} ; \quad \nu=\Phi(z)
$$

such that $\operatorname{ord}_{0}(\Phi)=b \cdot l \geq-n l+1$ and $n=c \cdot k$ for some integers $b, c$. Now

$$
w_{1}=z^{n} ; \quad w_{2}=\left(z^{n l} \cdot \Phi(z)\right)^{\frac{1}{k}}=\left(z^{n+b} \cdot f_{0}(z)\right)^{\frac{l}{k}} ; \quad f_{0}(0) \neq 0
$$

implies that $w_{2}$ is a multi-valued function of $z$. Subdivide the disc $D$ into equal sectors $Q_{m}, 0 \leq m \leq n-1$, and hence define on each $Q_{m}$ a holomorphic function $F_{m}(z)$, such that $F_{0}$ is the principal branch of $\left(z^{n+b} \cdot f_{0}(z)\right)^{\frac{l}{k}}$, and $F_{m+1}(z):=$ $e^{2 \pi i \frac{l}{k}} \cdot F_{m}(z)$.

As in the previous example, letting $\vartheta=2 \pi t$, we have

$$
\omega=\frac{1}{2}\left(f_{1}+i f_{2}\right)(\nu, \bar{\nu}) d \bar{\nu}=\frac{1-p \cdot \varphi(|\nu|)^{2}}{4 \pi l \cdot|\nu|^{2}} \cdot \boldsymbol{i} \nu d \bar{\nu}=\bar{\partial} g(\nu, \bar{\nu}) .
$$

Let

$$
\gamma(s):=\frac{1-p \cdot \varphi(\sqrt{s})^{2}}{l \cdot s},
$$

noting $\varphi(0)=\frac{1}{\sqrt{p}}$ implies that the improper integral

$$
\hat{\gamma}(s):=\int_{0}^{s} \gamma(\tau) d \tau
$$

is convergent. It follows that we can set $g(\nu, \bar{\nu})=\frac{\boldsymbol{i}}{4 \pi} \hat{\gamma}\left(|\nu|^{2}\right)$. Hence

is smoothly defined on $D$. Moreover

$$
\Phi^{*} \omega=\bar{\partial}(g \circ \Phi)=\frac{i}{4 \pi} \bar{\partial} \hat{\gamma}\left(|\Phi|^{2}\right)
$$

$$
\hat{G}=2 \pi \boldsymbol{i} g \circ \Phi=-\frac{1}{2} \hat{\gamma}\left(|\Phi|^{2}\right) .
$$

Setting $t=\frac{1}{2 \pi} \arg \left(z^{n}\right)$, we then have

$$
t+\boldsymbol{i} \hat{a}=\frac{1}{2 \pi \boldsymbol{i}}\left(\log \left(z^{n}\right)-\hat{G}\right)=\frac{1}{2 \pi} \arg \left(z^{n}\right)+\frac{\boldsymbol{i}}{2 \pi}(\hat{G}-n \ln |z|),
$$

with which we combine the statement of Theorem 1 to conclude

$$
a(z)=-n \ln \left(|z| e^{\frac{1}{2 n}} \hat{\gamma}\left(|\Phi|^{2}\right)\right)
$$

in order to define a pseudoholomorphic map $(\psi, a): D \backslash\{0\} \rightarrow \mathbb{E}_{p, q} \times \mathbb{R}$ of charge $n$ at the origin, asymptotic to the orbit corresponding to $\left\{w_{2}=0\right\}$.

\section{REFERENCES}

[1] Bourgeois, F. Ph.D. Thesis (preprint) Stanford 2004

[2] Brieskorn, E. and Knörrer, H. Plane Algebraic Curves, Birkhäuser 1986 MR886476 (88a:14001)

[3] Eliashberg, Y. Invariants in Contact Topology, Proceedings ICM, Berlin 1998, Volume II, Documenta Mathematica (1998) 327-338 MR1648083 (2000a:57068)

[4] Gluck. H. and Warner, F., Great Circle Fibrations of the Three-Sphere, Duke Math. J. 50 No.1 (1983) 107-132 MR700132 (84g:53056)

[5] Hale, J. Ordinary Differential Equations, Pure and Appl. Math. 21, Wiley 1969 MR0419901 (54:7918)

[6] Hofer, H., Pseudoholomorphic Curves in Symplectisations with applications to the Weinstein Conjecture in dimension three, Invent. Math. 114 (1993) 515-563 MR1244912 (94j:58064)

[7] Hofer, H. and Kriener, M. Holomorphic Curves in Contact Dynamics, Proc. Symp. Pure Math. 65 (1999) 77-131 MR1662750 (2000b:53113) 
[8] Hofer, H., Wysocki, K. and Zehnder, E. Properties of Pseudoholomorphic Curves in Symplectisations I: Asymptotics, Ann. Inst. Henri Poincaré 13 (1996) 337-379 MR1395676 (97e:58029)

[9] Hofer, H., Wysocki, K. and Zehnder, E. Properties of Pseudoholomorphic Curves in Symplectisations II: Embedding controls and algebraic invariants, Geom. Funct. Anal. 5 (1995) 270-328 MR1334869 (97e:58030)

[10] Hofer, H., Wysocki, K. and Zehnder, E. Properties of Pseudoholomorphic Curves in Symplectisations IV: Asymptotics with degeneracies, in "Contact and Symplectic Geometry", C.B. Thomas ed., Cambridge (1996) 78-117 MR1432460 (98e:58030)

[11] Martinet, J. Formes de Contact sur les variétés de dimension 3, Springer Lecture Notes 207 (1971) 142-163

[12] McDuff, D. Singularities of J-holomorphic Curves in almost complex 4-manifolds, J. Geom. Anal. 2 (1992) 249-266 MR1164604 (93g:58032)

[13] McKay, B. Dual Curves and Pseudoholomorphic Curves, Selecta Math. 9 (2003) 251-311 MR1993485 (2004g:53099)

[14] Micallef, M. and White, B. The structure of branch points in Minimal Surfaces and in Pseudoholomorphic Curves, Ann. Math. 139 (1994) 35-85 MR1314031 (96a:58063)

[15] Sikorav, J.-C. Singularities of J-holomorphic Curves, Math. Z. 226 (1997) 359-373 MR1483537 (98k:58060)

School of Mathematics, Statistics and Computer Science, University of New England, Armidale, NSW 2351, Australia

E-mail address: adamh@turing.une.edu.au

School of Mathematics and Statistics, Melbourne University, Parkville, ViC 3010, Australia

E-mail address: wysocki@ms.unimelb.edu.au 